\title{
Implementing Efficient All Solutions SAT Solvers
}

TAKAHISA TODA, University of Electro-Communications

TAKEHIDE SOH, Kobe University

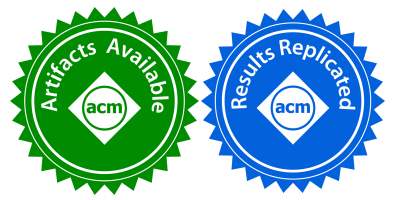

All solutions SAT (AllSAT for short) is a variant of the propositional satisfiability problem. AllSAT has been relatively unexplored compared to other variants despite its significance. We thus survey and discuss major techniques of AllSAT solvers. We accurately implemented them and conducted comprehensive experiments using a large number of instances and various types of solvers including a few publicly available software. The experiments revealed the solvers' characteristics. We made our implemented solvers publicly available so that other researchers can easily develop their solvers by modifying our code and comparing it with existing methods.

CCS Concepts: - Mathematics of computing $\rightarrow$ Combinatorial algorithms; $\bullet$ Theory of computation $\rightarrow$ Constraint and logic programming; $\bullet$ Software and its engineering $\rightarrow$ Constraints;

Additional Key Words and Phrases: AllSAT solvers, model enumeration, BDD, blocking clause, formula caching, CNF to DNF conversion, knowledge compilation, conflict-directed backjumping

ACM Reference Format:

Takahisa Toda and Takehide Soh. 2016. Implementing efficient all solutions SAT solvers. J. Exp. Algorithmics 21, 1, Article 1.12 (October 2016), 44 pages.

DOI: http://dx.doi.org/10.1145/2975585

\section{INTRODUCTION}

Propositional satisfiability (SAT for short) is the problem of determining whether a Boolean formula is satisfiable. SAT is ubiquitous in computer science. It has attracted the attention of many researchers from theory to practice because of its significance. Many applications have motivated empirical studies, in particular the development of SAT solvers, that is, software to solve satisfiability. A fundamental task of SAT solvers is to solve as many instances as possible in a realistic amount of time. Various practical algorithms and elegant implementation techniques have been developed [van Harmelen et al. 2007, Chap. 2; Biere et al. 2009, Chap. 4; Malik and Zhang 2009] to achieve this end.

There are many variants of SAT. We study All solutions SAT (AllSAT for short), or model enumeration. It is, given a Conjunctive Normal Form (CNF) formula, to generate partial satisfying assignments such that they form a logically equivalent Disjunctive Normal Form (DNF) formula. AllSAT has been relatively unexplored, compared to neighboring areas. Although this was reported in the literature [Jabbour et al. 2014a] and also is supported by the fact that there have only been a few recent papers, almost

This work is supported by JSPS KAKENHI Grant Number 26870011.

Authors' addresses: T. Toda, Graduate School of Informatics and Engineering, University of ElectroCommunications, 1-5-1 Chofugaoka, Chofu, Tokyo 182-8585, Japan; email: todat@acm.org; T. Soh, Information Science and Technology Center, Kobe University, 1-1 Rokkodai, Nada Kobe 657-8501, Japan; email: soh@lion.kobe-u.ac.jp.

Permission to make digital or hard copies of all or part of this work for personal or classroom use is granted without fee provided that copies are not made or distributed for profit or commercial advantage and that copies bear this notice and the full citation on the first page. Copyrights for components of this work owned by others than the author(s) must be honored. Abstracting with credit is permitted. To copy otherwise, or republish, to post on servers or to redistribute to lists, requires prior specific permission and/or a fee. Request permissions from permissions@acm.org.

2016 Copyright is held by the owner/author(s). Publication rights licensed to ACM.

ACM 1084-6654/2016/10-ART1.12 $\$ 15.00$

DOI: http://dx.doi.org/10.1145/2975585 
no software has been publicly available. ${ }^{1}$ Further, it has not even been taken up in major handbooks related to satisfiability.

A recent application of AllSAT is data mining. A fundamental task in data mining is to generate all interesting patterns from a given database [Agrawal et al. 1993; Agrawal and Srikant 1994; Han et al. 2007]. Examples include frequent itemsets, maximal frequent itemsets, or closed itemsets in transaction databases. Although algorithms for generating various patterns have been proposed, they are basically specialized for their target patterns. This means that different patterns require new algorithms. A framework based on a declarative paradigm has recently been proposed [Guns et al. 2011] for this reason. The basic flow is that constraints on patterns to be generated are formulated as logical formulas and solved with a generic solver. Hence, all that users do is simply model their problems, and they do not need to design algorithms. An approach on which problems are encoded into CNF formulas and solved with AllSAT solvers has been pursued [Jabbour et al. 2013] in much related work. An advantage of the declarative paradigm is its ability to flexibly handle new patterns. There is no need to examine details of algorithms on which solvers are based; thereby, it is open to a wider range of users. However, it is inferior in efficiency to problem-specific approaches. It is necessary, in practice, to balance between efficiency and flexibility. Therefore, improving solver's performance is essential in the declarative framework.

Apart from data mining, there are many studies on the application of AllSAT, particularly to formal verification, such as network verification [Zhang et al. 2012; Lopes et al. 2013; Qadir and Hasan 2015; Lopes et al. 2015; Jayaraman et al. 2014], predicate abstraction [Clarke et al. 2004; Lahiri et al. 2003, 2006], and image and preimage computation in unbounded model checking [Gupta et al. 2000; McMillan 2002; Sheng and Hsiao 2003; Li et al. 2004; Ganai et al. 2004; Grumberg et al. 2004].

We found it was important to clarify state-of-the-art techniques of AllSAT solvers by considering these studies and to improve them on a firm basis. However, there were three issues in existing research on AllSAT.

- Several methods have been proposed but they have not been globally compared. It is thus difficult to determine which method is effective for what kinds of AllSAT instances.

-Experiments have not been carried out on comprehensive benchmarks.

- There have been few public AllSAT solvers, which makes it difficult to compare existing techniques.

Here, we would thus like to survey major techniques of AllSAT solvers and try to complement past references by gathering and organizing existing techniques. We further add some novel techniques. We conduct experimental comparisons, to evaluate solvers, including clasp, which is one of the few off-the-shelf software available with solution enumeration support. We make our implemented solvers publicly available from our website $^{2}$ with expectations that further improvements to solvers and their evaluations can easily be done and AllSAT research will be stimulated.

The article is organized as follows. Section 2 provides related work on AllSAT. Section 3 provides necessary notions, terminology, and results. Section 4 surveys major techniques of AllSAT solvers, where we indicate those including our original ideas

\footnotetext{
${ }^{1} \mathrm{~A}$ few exceptions are clasp [Gebser et al. 2007], picosat [Biere 2008], and relsat [Bayardo, Jr. and Pehoushek 2000]. Although they support solutions enumeration, they are positioned as an answer set solver, a SAT solver, and a \#SAT solver, respectively, rather than AllSAT solvers.

${ }^{2}$ All Solutions SAT Repository by T. Toda, http://www.sd.is.uec.ac.jp/toda/code/allsat.html. It was accessed May 16, 2016.
} 
by adding asterisks to their titles. Section 5 provides experimental results. Section 6 concludes the article.

\section{RELATED PROBLEMS}

Another variant of SAT is the dualization of Boolean functions; given a DNF formula of a Boolean function, $f$, it is the problem of computing the complete DNF formula of the dual function, $f^{d}$. Since a CNF formula of $f^{d}$ can easily be obtained by interchanging a logical disjunction with a logical conjunction as well as the constants 0 with 1 , the main part is to convert CNF to the complete DNF. Hence, the essential difference from AllSAT is that the resulting DNF formula must be complete. Dualization has been well studied in terms of complexity [Eiter et al. 2008; Crama and Hammer 2011], while to the best of our knowledge, there have been few recent empirical studies on it [Jin and Somenzi 2005; Brauer et al. 2011; Jabbour et al. 2014b; Toda 2016]. Practical algorithms for a restricted form of dualization have been presented [Toda 2013; Murakami and Uno 2014; Gainer-Dewar and Vera-Licona 2016] and some implementations are available, ${ }^{3}$ although they are not for arbitrary Boolean functions.

Another variant is the problem of counting the number of total satisfying assignments, called propositional model counting, or \#SAT, which has been well studied [Biere et al. 2009, Chap. 20]. There are important applications such as those for probabilistic inference problems and hard combinatorial problems. Several solvers, useful tools such as a preprocessor [Lagniez and Marquis 2016], and benchmarks are publicly available in BeyondNP.org. ${ }^{4}$ Although \#SAT is apparently similar to AllSAT, techniques such as connected components and component caching are inherent in counting, and they are not applicable to AllSAT as they were in Morgado and MarquesSilva [2005].

\section{PRELIMINARIES}

Necessary notions, terminology, and results concerning Boolean functions, satisfiability solvers, and binary decision diagrams are presented in this section.

\subsection{Boolean Basics}

A literal is a Boolean variable or its negation. A clause is a finite disjunction of literals, and a term is a finite conjunction of literals. A propositional formula is in conjunctive normal form (CNF for short) if it is a finite conjunction of clauses and in disjunctive normal form ( $D N F$ for short) if it is a finite disjunction of terms. We identify clauses with sets of literals and CNF formulas with sets of clauses. The same applies to terms and DNFs. Boolean formulas are logically equivalent if they represent identical Boolean functions.

An assignment to a set $V$ of Boolean variables is a partial function from $V$ to $\{0,1\}$. A satisfying assignment for a CNF formula is an assignment $v$, such that the CNF formula evaluates to 1 . An assignment to $V$ is total (or complete) if it is a total function, that is, all variables in $V$ are assigned values. A Boolean formula is satisfiable if it has a satisfying assignment. For simplicity, we say that a literal is assigned value $v$ if the

\footnotetext{
${ }^{3}$ The Hypergraph Dualization Repository by K. Murakami and T. Uno, http://research.nii.ac.jp/ $\sim$ uno/dualization.html. It was accessed Jan. 19, 2013. The Hypergraph Transversal Computation with Binary Decision Diagrams (HTC-BDD) by T. Toda. This work was done at the ERATO MINATO Discrete Structure Manipulation System Project of the Japan Science and Technology Agency at Hokkaido University, http://www.sd.is.uec.ac.jp/toda/htcbdd.html. It was accessed Sept. 10, 2015. MHS generation algorithms by Vera-Licona's Research Group, https://github.com/VeraLiconaResearchGroup/MHSGenerationAlgorithms. It was accessed May 20, 2016.

${ }^{4}$ BeyondNP.org, hosted by the Automated Reasoning Group at the University of California, Los Angeles. http://beyondnp.org/pages/tools/. It was accessed Jul. 27, 2016.
} 


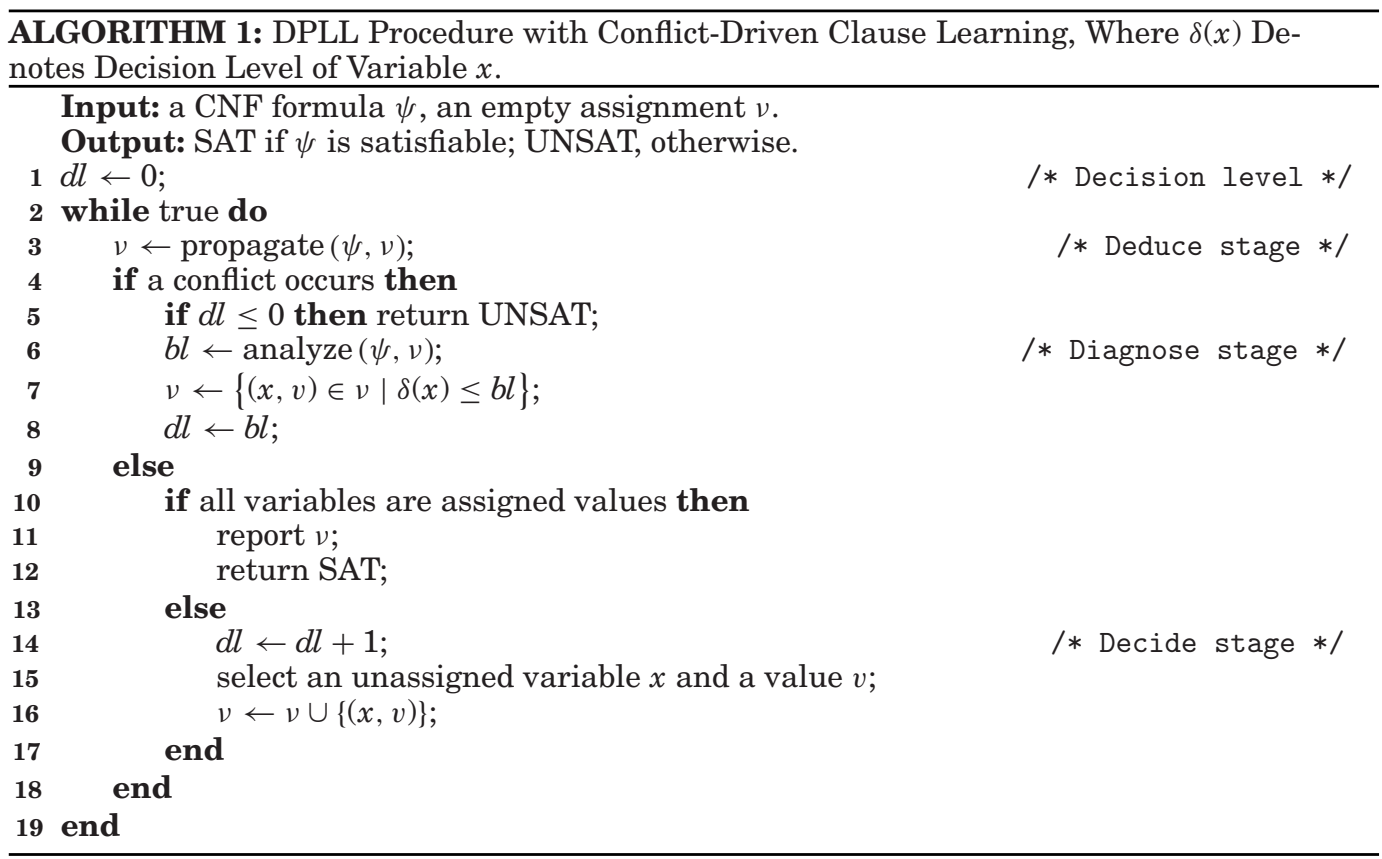

assignment to the underlying variable makes the literal evaluate to $v$. If there are no concerns about confusion, we identify an assignment function, $v$ over $V$, with the set of the form $\{(x, v) \in V \times\{0,1\} \mid v(x)=v\}$. We also identify the assignment, $x \mapsto v$, with $x$ if $v=1$ and with $\neg x$ if $v=0$. Assignments and literals are used interchangeably in this way throughout the article.

Example 3.1. Consider the sequence of literals $x_{1}, \neg x_{5}, x_{3}$. This means that $x_{1}, x_{5}, x_{3}$ are selected in this order and the values $1,0,1$ are respectively assigned.

The All Solutions SAT Problem (AllSAT for short) is defined as follows: given a CNF, $\psi$, compute satisfying assignments for $\psi$ such that the DNF consisting of the term representations of these assignments represents the same Boolean function as $\psi$. Note that each computed assignment function, $v: V \rightarrow\{0,1\}$, is identified with the conjunction of literals that correspond to assignments $v(x)$ to defined variables $x \in V$. Satisfying assignments may be partial or total. There are numerous DNF representations for an identical Boolean function, meaning that different AllSAT solvers may compute syntactically different but logically equivalent DNF formulas.

\subsection{Satisfiability Solvers}

The propositional satisfiability problem (SAT for short) is the problem of deciding whether there is a satisfying assignment for a CNF formula. Algorithm 1 indicates the basic framework on which modern SAT solvers are based. Other techniques such as lazy data structures, variable selection heuristics, restarting, and deletion policies of learnt clauses have been omitted for simplicity (see van Harmelen et al. [2007, Chap. 2], Biere et al. [2009, Chap. 4], and Knuth [2011] for details).

The basic behavior of Algorithm 1 is to search satisfying assignments in such a way that a solver extends a candidate assignment by assigning values to variables and if the assignment turns out to be unsatisfying, the solver proceeds to the next candidate by backtracking. The extension of an assignment is triggered at the decide stage, where 
an unassigned variable, $x$, is selected and a value, $v \in\{0,1\}$, is assigned to $x$; it is then spread at the deduce stage, where assignments to other variables are deduced from the most recent decision, $(x, v)$.

Decision assignments are those given at the decide stage and decision variables are those assigned values there. Consider a decision tree that branches at each decision assignment. A decision level is the depth of that decision tree, which is maintained by the variable $d l$ in Algorithm 1. The decision level of a variable, $x$, is denoted by $\delta(x)$, which is the one at which $x$ is assigned a value. For a literal, $l$, the notation $\delta(l)$ is defined as that of the underlying variable, and $l @ d$ denotes that $l$, seen as an assignment, is given at level $d$, that is, $\delta(l)=d$.

The deduce stage is described in the following. A clause is a unit clause if all but one of the literals are assigned value 0 and the remaining one is unassigned. The remaining literal is called a unit literal. Unit clauses are important in the deduce stage because assignments to the underlying variables of unit literals are necessarily determined so that unit literals evaluate to 1 . After some assignments are determined by unit clauses, nonunit clauses may become unit clauses. Hence, all implications are deduced until there is a falsified clause or no unit clause exists. This process is called unit propagation. The function, propagate, performs unit propagation. Implied assignments are those given at this stage and implied variables are those assigned values there. The notion of the decision levels of implied variables $x$, the notations $\delta(x)$ and $l @ d$, where $l$ is a literal representing an implied assignment, are defined in the same way.

Example 3.2. Consider the CNF formula, $\psi$, that consists of the following clauses.

$$
\begin{aligned}
& C_{1}=x_{1} \vee \neg x_{3}, \\
& C_{2}=x_{2} \vee x_{3} \vee x_{5}, \\
& C_{3}=\neg x_{1} \vee \neg x_{3} \vee x_{4}, \\
& C_{4}=x_{4} \vee \neg x_{5} \vee x_{6}, \\
& C_{5}=x_{5} \vee \neg x_{6} .
\end{aligned}
$$

Assume the decision assignment, $\neg x_{5} @ 1$. The implied assignment, $\neg x_{6} @ 1$, is obtained from $C_{5}$. Assume the decision assignment, $x_{3} @ 2$. The implied assignments, $x_{1} @ 2$ and $x_{4} @ 2$, are obtained from $C_{1}$ and $C_{3}$ in this order. Assume the decision assignment, $x_{2} @ 3$; the $\mathrm{CNF}$ formula is then satisfied.

It should be noted that we may identify a falsified clause in the middle of unit propagation. This case is called a conflict. As soon as a conflict occurs, unit propagation halts, even though unit clauses still remain. In the case of conflict, if all assigned variables are those assigned prior to any decision (i.e., $d l=0$ ), it means that there are no other assignments to be examined; thereby, a CNF formula must be unsatisfiable. In that case, a solver halts, reporting UNSAT. If a decision has been made at least once, we enter into the diagnose stage to resolve conflict.

The "cause" of the conflict we have just encountered is analyzed at the diagnose stage; a new clause is learnt as a result, and it is added to the CNF formula, by which a solver is guided not to reenter the conflict (and other conflicts related to it). Modern solvers maintain an implication graph during search to do this efficiently, which represents an implication relation between assignments over unit propagation. More specifically, an implication graph is a directed acyclic graph, $G=(V, A)$, such that:

-vertices in $V$ correspond to literals, $l$, representing assignments to their underlying variables; 


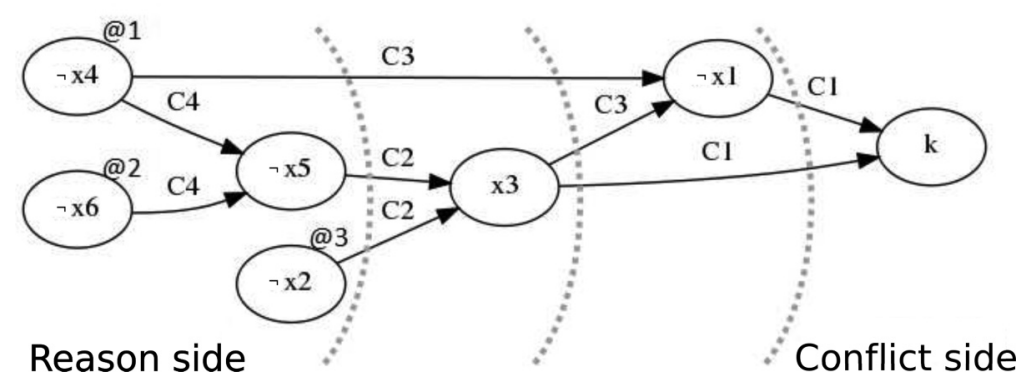

Fig. 1. Conflict graph, where arcs are labeled with antecedents of their target vertices.

- $\operatorname{arcs}$ in $A$ correspond to implications so that if there is a unit clause, $C=\left\{l_{1}, \ldots, l_{n}\right\}$, with $l_{1}$ to $l_{n-1}$ all evaluated to 0 , then there is an arc from each literal $\neg l_{i}(1 \leq i \leq n-1)$ to $l_{n}$, meaning that the assignment that makes $l_{n}$ evaluate to 1 is implied by $\bar{C}$; and -if there is a falsified clause, $C=\left\{l_{1}, \ldots, l_{n}\right\}$, then there is an arc from each literal $\neg l_{i}(1 \leq i \leq n)$ to the special vertex, $\kappa$.

An implication graph might be implemented so that whenever a variable, $x$, is implied, it is associated with the clause that determines the assignment to $x$ as a unit clause. This clause is called the antecedent of $x$.

Example 3.3. Consider the CNF formula, $\psi$, defined in Example 3.2. Assume in turn the decision assignments, $\neg x_{4} @ 1, \neg x_{6} @ 2$, and $\neg x_{2} @ 3$, in this order. The resulting implication graph is outlined in Figure 1 . This case results in a conflict because $C_{1}$ becomes falsified.

A conflict graph is a subgraph, $H$, of an implication graph, $G$, obtained by restricting $G$ so that all vertices have paths to $\kappa$. For a subset, $U$, of vertices in a conflict graph, $H=(V, A)$, the arc-cut (called cut after this) corresponding to $U$ is the set of arcs that connects vertices in $U$ with those in $V \backslash U$. Examples are indicated by the dotted curves in Figure 1.

We are now ready to describe a clause learning scheme, which is performed by the function, analyze. Consider cuts such that all decision assignments are on one side, called the reason side, and the special vertex, $\kappa$, is on the other side, called the conflict side. Take the negation of literals on the reason side that are incident to arcs in a cut, which form a conflict-driven clause (or a conflict clause). This clause is considered to be a "cause" of conflict. Indeed, if variables are assigned values according to the literals on the reason side that are incident to arcs in a cut, then the same implications on the conflict side are derived and the same conflict must occur. Therefore, it is necessary for variables to be assigned values so that at least one of these literals is negated to avoid the conflict. This condition is formulated as the conflict clause described previously.

As we can see from Figure 1, there are many choices for cuts that induce conflict clauses. Of these, conflict clauses that contain exactly one literal from the current decision level are known to be effective. A Unique Implication Point (UIP for short) is a vertex in a conflict graph such that every path from the decision at the current decision level to $\kappa$ passes through it. Note that there is at least one UIP because the decision at the current decision level is a UIP. The first UIP scheme is to find the UIP that is closest to $\kappa$.

Example 3.4. Consider the conflict graph in Figure 1. The middle curve gives the first UIP, $x_{3}$. Hence, the conflict clause is $x_{4} \vee \neg x_{3}$. 
The first UIP scheme can be efficiently carried out by traversing a conflict graph from $\kappa$ in the reverse order of implications, based on the implementation of the implication graph stated previously. During traversal, it is easy to decide whether the current literal is a UIP. Consider the cut that induces a UIP. The assignments for all literals on the reason side are determined prior to those on the conflict side. Since the traversal is in the reverse order of implications, the UIP can then be located by keeping track of the number of unvisited vertices of the current decision level that are immediate neighbors of visited vertices.

All that remains is to decide a backtrack level, $b l$, cancel all assignments above $b l$, and set $b l$ to the current decision level. There are two choices to decide a backtrack level. Assume that the decision, $p$, at level $i$ results in a conflict and there is no solution that extends the current assignment. Chronological backtracking cancels all assignments of level $i$ including decision $p$ and attempts to find a solution by extending the assignment from level $i-1$ with a conflict clause. Chronological backtracking undoes assignments from a higher to a lower level in this way. A drawback is that if there is no solution in higher levels, it is hard to leave those levels. Nonchronological backtracking, on the other hand, immediately jumps to a lower level, $j$, by canceling all assignments above $j$ and attempts to find a solution upward by extending the assignment from level $j$. The backtrack level, $j$, is commonly determined as the greatest level of a variable in a conflict clause below the current level, $i$. For each canceled assignment between levels $j$ and $i$, the possibility of becoming a solution is generally left, although this does not mean that a solver loses the opportunity to find solutions.

Example 3.5. Consider the conflict graph in Figure 1. For the conflict clause $x_{4} \vee \neg x_{3}$, the nonchronological backtrack level is 1 . Hence, the assignments, $\neg x_{6} @ 2, \neg x_{5} @ 2$, are canceled and search restarts from $\neg x_{4} @ 1$; however, a solution could be obtained by backtracking to level 2 .

\subsection{Binary Decision Diagrams}

A Binary Decision Diagram ( $B D D$ for short) is a graphical representation of Boolean functions in compressed form [Lee 1959; Akers 1978; Bryant 1986]. We follow the notation and terminology in Knuth [2011, Chapter 7.1.4].

Figure 2 has an example of a BDD. Exactly one node has indegree 0, which is called the root. Each branch node, $f$, has a label and two children. Node labels are taken from variable indices, and the children consist of the LO child and the HI child. The arc to a LO child is called a LO arc, indicated by the dotted arrow, and the LO arc of $f$ means value 0 is assigned to the variable of $f$. Similarly, the arc to a HI child is called a HI arc, indicated by the solid arrow, and the HI arc of $f$ in turn means value 1 is assigned to its variable. There are two sink nodes that are denoted by $T$ and $\perp$. Paths from the root to $T$ and $\perp$ mean satisfying assignments for the former and unsatisfying assignments for the latter.

BDDs are called ordered if for any node, $u$, with a branch node, $v$, as its child, the index of $u$ is less than that of $v$. BDDs are called reduced if the following two reduction operations cannot be applied further.

(1) If there is a branch node, $u$, whose arcs both point to $v$, then redirect all the incoming arcs of $u$ to $v$, and then eliminate $u$ (Figure 3(a)).

(2) If there are two branch nodes, $u$ and $v$, such that the subgraphs rooted by them are equivalent, then merge them (Figure 3(b)).

Ordered reduced BDDs in this article are simply called BDDs. Ordered BDDs that do not need to be fully reduced are distinguished from ordinary BDDs by calling them $O B D D s$. Note that each node in a BDD (or an OBDD) is conventionally identified with the subgraph rooted by it, which also forms a BDD (or an OBDD). 


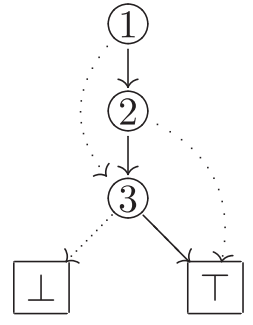

Fig. 2. BDD representation for function $f\left(x_{1}, x_{2}, x_{3}\right)=x_{1} \wedge \neg x_{2} \vee x_{3}$.

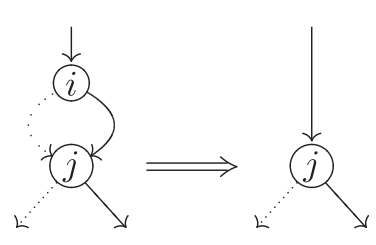

(a) Node elimination

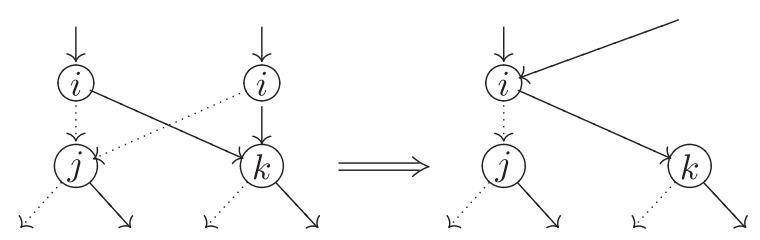

(b) Node merging

Fig. 3. Reduction rules.

\section{TECHNIQUES OF ALL SOLUTIONS SAT SOLVERS}

We must carefully determine an appropriate suite of techniques by considering various factors and their characteristics to implement an efficient AllSAT solver. Some details have only been partly explained and scattered in past references. We thus survey major existing techniques of AllSAT solvers and try to complement past references to these by gathering and organizing existing techniques. We also add some novel techniques.

This section is organized as follows. Three major types of solvers are presented in their own subsections. Each subsection starts with an overview, then provides specific techniques, and ends with the configurations for our implemented solvers. We add an asterisk to the title of each specific technique that contains our original ideas.

\subsection{Blocking Solvers}

4.1.1. Overview. One of the easiest ways of implementing an AllSAT solver is to repeatedly run an ordinary SAT solver as a black box and find satisfying assignments one-by-one. One specific procedure is detailed next.

(1) Run a SAT solver with a CNF formula, $\psi$.

(2) If $\psi$ is unsatisfiable, halt.

(3) Report a found total satisfying assignment, $v$.

(4) Compute the clause, $C$, of the form $\{x \mid v(x)=0\} \cup\{\neg x \mid v(x)=1\}$, in a set notation.

(5) Add $C$ to $\psi$ and go to Step 1.

The clause obtained in Step 4 is called a blocking clause [McMillan 2002]. Since a blocking clause, $C$, is the complement of the term corresponding to the current satisfying assignment, $v$, the extended CNF formula, $\psi \cup\{C\}$, is falsified by $v$ in a later search; thereby, a new solution will be found in each iteration. Furthermore, since $v$ is a total assignment, no assignments other than $v$ are blocked.

Example 4.1. Execute the procedure with the CNF formula:

$$
\psi=\left(x_{1} \vee \neg x_{2}\right) \wedge\left(x_{2} \vee \neg x_{3}\right) \wedge\left(x_{3} \vee \neg x_{1}\right) .
$$

Suppose a solver returns the satisfying assignment, $\neg x_{1}, \neg x_{2}, \neg x_{3}$. The blocking clause, $x_{1} \vee x_{2} \vee x_{3}$, is added to $\psi$ :

$$
\psi=\left(x_{1} \vee \neg x_{2}\right) \wedge\left(x_{2} \vee \neg x_{3}\right) \wedge\left(x_{3} \vee \neg x_{1}\right) \wedge\left(x_{1} \vee x_{2} \vee x_{3}\right)
$$




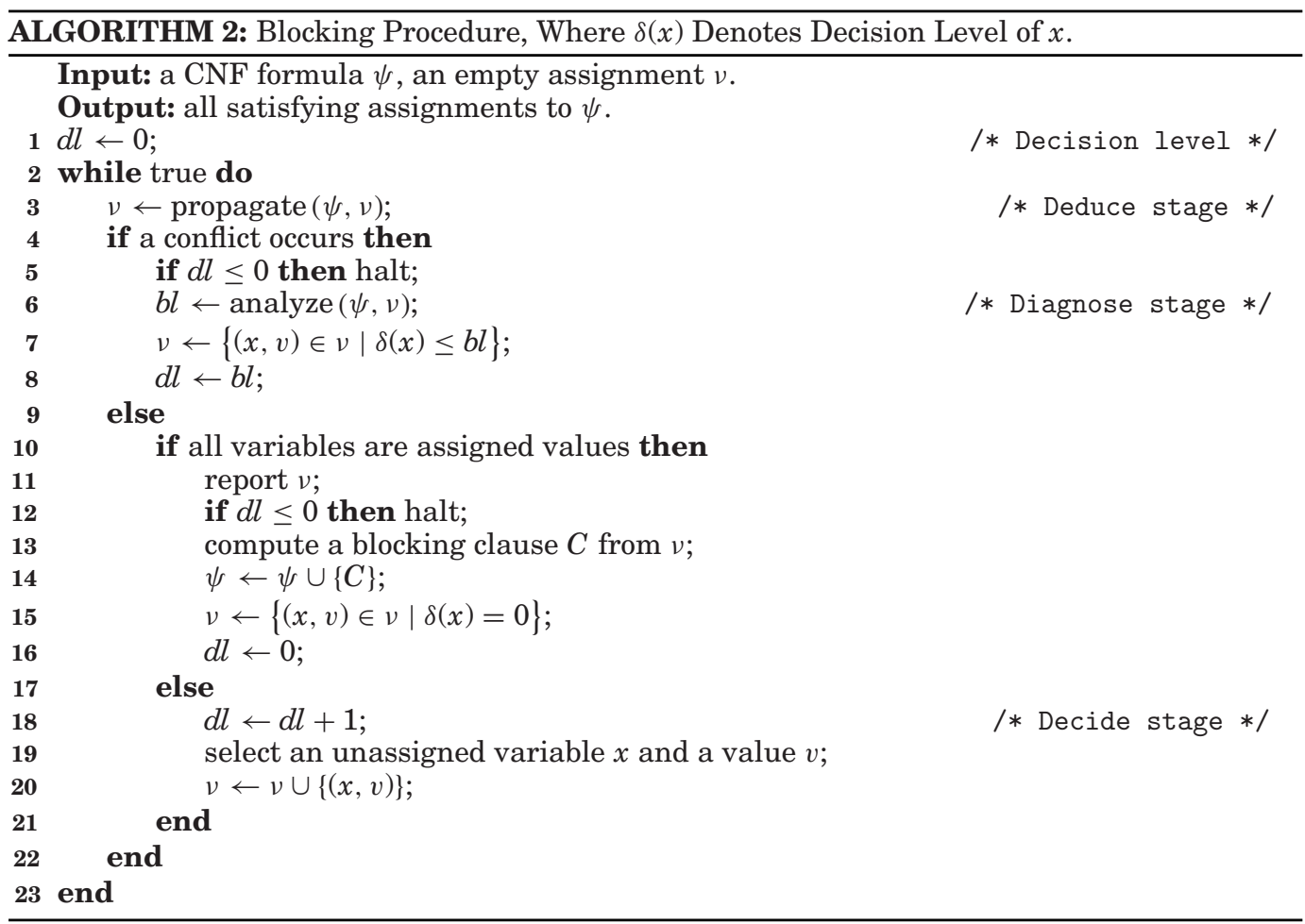

The solver then returns the satisfying assignment, $x_{1}, x_{2}, x_{3}$. The blocking clause, $\neg x_{1} \vee$ $\neg x_{2} \vee \neg x_{3}$, is added to $\psi$ :

$$
\psi=\left(x_{1} \vee \neg x_{2}\right) \wedge\left(x_{2} \vee \neg x_{3}\right) \wedge\left(x_{3} \vee \neg x_{1}\right) \wedge\left(x_{1} \vee x_{2} \vee x_{3}\right) \wedge\left(\neg x_{1} \vee \neg x_{2} \vee \neg x_{3}\right)
$$

This time a solver returns UNSAT, which means all satisfying assignments are found.

Since a blocking clause has a size equal to the number of variables, unit propagation is likely to slow down. Hence, it is arguably better to consider blocking clauses that only consist of decisions, which we call decision-based blocking clauses for convenience. Since decisions determine the other assignments, decision-based blocking clauses have the same effect as those of all literals. However, to know which literals are decisions, we need to modify a solver code. Algorithm 2 is the pseudocode obtained by modifying Algorithm 1. For convenience, we simply call this a blocking procedure to distinguish it from the other procedures that will be presented later. Only lines 5 and 12-16 are changed. A solver halts at lines 5 and 12 because $d l \leq 0$ means that all variables are implied without any decision and all solutions are found. All assignments except for those determined without any decision are canceled at line 15, and a solver backtracks to the root level at line 16.

A decision-based blocking clause only blocks a single assignment and there are as many blocking clauses as total satisfying assignments. Since they are stored, this is likely to result in space explosion and slow down unit propagation. This is considered a serious issue since unit propagation in modern SAT solvers occupies the majority of the entire processing time. Another disadvantage is that whenever a solution is found, a solver is forced to restart from scratch with an extended CNF formula, while not resuming search. 
The blocking clause-based implementation might be considered to be an advantageous choice for instances when even a single solution is hard to find, or for instances with a small number of solutions. It can easily be implemented because the blocking clause mechanism can be achieved outside a solver or with a small modification to the solver code, as demonstrated in Algorithm 2. We can also benefit from powerful techniques of modern SAT solvers, such as conflict-driven clause learning and nonchronological backtracking.

Remark 4.2. Once blocking clauses are added to a CNF formula, they are not deleted afterward and must not be treated in the same way as conflict clauses. Otherwise, solutions would be rediscovered many times, which was not allowed in the research discussed in this article.

4.1.2. Simplifying Satisfying Assignments. Simplification of satisfying assignments is to obtain from a satisfying assignment, $v$, to a CNF formula, $\psi$, a smaller assignment, $v^{\prime}$, that still makes $\psi$ evaluate to $1 .^{5}$ This is done by canceling assignments to redundant variables in $v$, where redundant means either value can be assigned without effect on the value of $\psi$. A simplified assignment is generally partial and it represents a set of total assignments, including the original assignment and possibly other satisfying assignments. Variable lifting refers to a number of such simplification techniques [Ravi and Somenzi 2004]. Since this topic has been well summarized in Morgado and Marques-Silva [2005], we will not go into the details here. Interested readers are referred to Morgado and Marques-Silva, as well as the references therein (see also Yu et al. [2014] for recent results).

Simplification allows us to obtain, from a single solution, possibly exponentially many solutions in compact form, that is, as a partial assignment. It is advantageous if we can obtain a partial assignment of minimum size; however, minimization is known to be computationally hard, and we must compromise, in practice, with near-minimum assignment by means of approximation. ${ }^{6}$ To combine simplification with the blocking mechanism of Algorithm 2, it suffices to perform simplification just before line 13 and then to take the complement of decisions in a simplified assignment. It should be noted that if a simplified blocking clause is empty, that is, all variables except for implied ones turn out to be redundant, then this means that all remaining solutions are covered. Thus, a solver in that case must be halted. The number of blocking clauses may be largely reduced due to simplification, which leads to a beneficial effect on unit propagation at the cost of performing simplification.

Example 4.3. Consider the CNF formula, $\psi$, defined in Example 3.2. The assignments, $\neg x_{5} @ 1, \neg x_{6} @ 1, x_{3} @ 2, x_{1} @ 2, x_{4} @ 2, x_{2} @ 3$, are given in this order. The last decision is redundant and by removing it, we obtain the partial assignment, $\neg x_{5} @ 1, \neg x_{6} @ 1, x_{3} @ 2, x_{1} @ 2, x_{4} @ 2$. Consider the decision assignments, $\neg x_{5} @ 1$ and $x_{3} @ 2$, by which the other variables are implied or can be assigned either value. By flipping these assignments, we obtain the simplified blocking clause, $C_{6}=x_{5} \vee \neg x_{3}$.

4.1.3. Continuing Search. Whenever a solution is found in Algorithm 2, a solver is forced to backtrack to the root level. After that, a different assignment will be examined due to a variable selection heuristic, and a region of the search space in which a solution was just found remains incomplete, which may result in unnecessary propagations and conflicts in a later search for that region. Restart is, however, essential in AllSAT

\footnotetext{
$\overline{5}$ We say that an assignment, $v^{\prime}$, is smaller than $v$ if all variables $x$ assigned values in $v^{\prime}$ are also assigned values in $v$ and their values coincide, that is, $v(x)=v^{\prime}(x)$.

${ }^{6}$ Even computing a minimal satisfying assignment requires quadratic time, which is still computationally expensive.
} 
solving, particularly when simplification is used. Indeed, the state of implications, such as which literals are decisions, becomes inconsistent, and it is necessary to again deduce implications after simplification is carried out and a new clause is added. There is no straightforward way to answer how to continue search [Jin et al. 2005].

The problem of overcanceling due to backtracking has been addressed [Pipatsrisawat and Darwiche 2007], and a simple technique called progress saving that stores recently canceled decisions in an array and simulates them after backtracking has been proposed. Any time a solver specifically enters into the decide stage, it checks whether an assignment to a selected variable is stored, and it simulates the previous decision if it exists; otherwise, it follows a default heuristic. Although this technique has been proposed within the context of SAT, it can also be applied to AllSAT.

Example 4.4. Continuing from Example 4.3, suppose that we add the blocking clause, $C_{6}$, to $\psi$ and backtrack to the root level with progress saving enabled. At this point, all assignments above the root level are canceled, yet the previous decisions, $\left(x_{5}, 0\right),\left(x_{3}, 1\right)$, are stored in an array. If $x_{5}$ or $x_{3}$ is selected, the previous decision is made again.

4.1.4. Implementation. We implemented four programs based on a blocking procedure according to whether simplification and continuation techniques were selected or not.

Our simplification method is related to the set covering model [Morgado and Marques-Silva 2005] and a decision-based minimal satisfying cube [Yu et al. 2014]. For the sake of efficiency, near-minimal satisfying assignments are computed. The basic idea is that given a total assignment, we select a small number of decision variables that make a CNF formula evaluate to 1 . This is done in two ways:

(1) Select all decision variables that relate to implications of at least one variable.

(2) For each clause that is not satisfied by selected variables, select an arbitrary decision variable that makes the current clause satisfied.

A simplified satisfying assignment then consists of assignments to the selected decision variables and all implied variables. By flipping the assignments to the selected decisions, we obtain a blocking clause, which blocks all total assignments represented by the simplified assignment.

We implement a continuation technique so that before backtracking at line 15, all decisions are stored in an array and after backtracking, we simulate these decisions whenever possible in the order of their decision levels. Clearly, not all decisions are simulated because of the existence of the blocking clause, and conflict or contradiction with the previous decision will occur. It is at this point where a solver continues search, and we will then enter into the conflict resolution or the decide stage.

\subsection{Nonblocking Solvers}

4.2.1. Overview. We will now provide a basic overview of the AllSAT procedure without the aid of blocking clauses. We modify Algorithm 1. The main feature is to employ chronological backtracking instead of nonchronological backtracking. The chronological backtracking we use is slightly different from the standard one described in Section 3.2. As indicated in Algorithm 3, the only difference is to insert the flipped decision, $(x, \bar{v})$, in $v$ and register it to an implication graph so that it has no incoming arc. This is because the implications of flipped decisions are not deduced without blocking clauses.

A literal, seen as an assignment, has a NULL antecedent if there is no implication for it: in other words, there is no incoming arc in an implication graph. The previous chronological backtracking is abbreviated as $B T$ after this for convenience, and it is performed by the function backtrack. We collectively call a number of procedures for AllSAT solving based on BT nonblocking procedures in contrast to the blocking 


\begin{tabular}{rrrr}
$-1^{*}$ & & & \\
\hline$-20^{*}$ & 19 & 9 & -15 \\
-8 & 12 & & \\
\hline$-18^{*}$ & 2 & -5 & -17 \\
-10 & 11 & -14 & 13 \\
-6 & -3 & 7 & 4 \\
$16_{*}$ & & &
\end{tabular}

(a) Solution Found

\begin{tabular}{rrrr}
$-1^{*}$ & & & \\
\hline$-20^{*}$ & 19 & 9 & -15 \\
-8 & 12 & $18_{*}$ & \\
\hline$-16^{*}$ & -6 & 11 & 2 \\
-7 & 13 & -5 & -3
\end{tabular}

(b) Conflict

\begin{tabular}{rrrr}
$-1^{*}$ & & & \\
\hline$-20^{*}$ & 19 & 9 & -15 \\
-8 & 12 & $18_{*}$ & 6 \\
-16 & -3 & &
\end{tabular}

(c) Conflict

Fig. 4. (a)-(c) Snapshots of nonblocking solver's state in three different cases. Assignments are given from left to right, top to bottom, separated by a line for each level. Integers $i$ specify assignments such that variable $x_{|i|}$ is assigned value 0 if $i<0$ and 1 if $i>0$. Decision assignments have asterisks as superscripts. Nondecision assignments with NULL antecedents have asterisks as subscripts. Instance used was uf20022.cnf, obtained from the Satisfiability Library (SATLIB), and the conflict resolution method used consisted of decision-level-based first UIP scheme and nonchronological backtracking with level limit, which will be introduced later.

ALGORITHM 3: Chronological Backtracking in Nonblocking Procedure, Where $\delta(y)$ Denotes Decision Level of $y$.

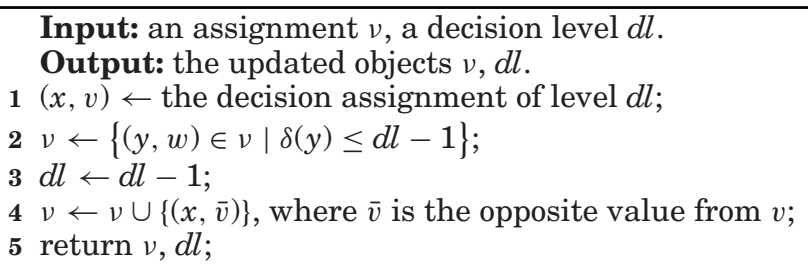

procedures presented in Section 4.1. If there are no concerns about confusion, chronological backtracking means BT.

An important concern in this approach is how to make BT compatible with conflictdriven clause learning. Consider a conflict graph in the clause learning phase. Due to BT, a conflict graph may contain several roots, that is, assignments with NULL antecedents at the same decision level (see, e.g., literals $\neg x_{20}$ and $x_{18}$ in Figure 5). Since an ordinary first UIP scheme commonly assumes a unique root at the same decision level, implementations based on that assumption get stuck in nondecision literals with NULL antecedents. Two techniques will be presented later to resolve this problem.

Example 4.5. If we look at Figure 4(a), all variables are assigned values without conflict, which means a solution is found. All assignments of level 3 in (a) are canceled and the flipped decision, $x_{18}$, is inserted in (b) as a nondecision assignment at level 2. Since no propagation takes place, a new decision, $\neg x_{16}$, is made and a subsequent propagation results in a conflict. The decision is the only one that has a NULL antecedent at the current decision level, and an ordinary first UIP scheme suffices in this case. The conflict clause, $x_{6} \vee x_{1} \vee \neg x_{18} \vee \neg x_{12}$, is then learnt from the conflict, and a solver backtracks to level $2 .^{7}$ The assignment, $x_{6}$, is implied in (c) by the conflict clause. A subsequent propagation results in a conflict again. This time, there is a nondecision assignment with a NULL antecedent at the same decision level.

A major advantage of the nonblocking approach is that no matter how many solutions exist, the performance of unit propagation does not deteriorate due to the absence of

\footnotetext{
${ }^{7}$ In this case, there is no opportunity of rediscovering solutions by ordinary chronological backtracking, and hence the flipped decision, $x_{16}$, is not inserted. This technique will be explained in more detail when nonchronological backtracking with a level limit is introduced.
} 
blocking clauses. Instead, it must find total satisfying assignments one-by-one. Hence, there is a limit to the number of solutions to be generated in a realistic amount of time.

4.2.2. Sublevel-Based First UIP Scheme. Grumberg et al. introduced the notion of sublevels and presented a sublevel-based first UIP scheme that was compatible with the nonblocking approach [Grumberg et al. 2004]. The basic idea is to divide a single decision level into sublevels. A new sublevel is specifically defined whenever BT is performed, and sublevels are undefined whenever their decision levels are undefined. An ordinary first UIP scheme is then able to be applied to the current sublevel.

Example 4.6. We will now illustrate how a decision level and a sublevel change by using Example 4.5. Let us start from the time just after a solution is found in (a). Since the decision, $\neg x_{18}$, is canceled, the decision level changes from 3 to 2 , and the sublevel also accordingly decreases by one. However, since the flipped decision, $x_{18}$, is then inserted, accompanied by BT, as a nondecision assignment with a NULL antecedent, the sublevel increases by one. Hence, assignments of decision level 2 that have been inserted before the present time have sublevel 2, while $x_{18}$ has sublevel 3 . After that, a new decision, $\neg x_{16}$, is made, by which the decision level and the sublevel increase by one, and their values correspond to 3 and 4 . The succeeding implied assignments cause a conflict in (b). After the conflict is resolved, the decision level changes from 3 to 2 , and the sublevel changes from 4 to 3 . Note that this case does not involve increments of sublevels since BT is not performed. ${ }^{8}$ The succeeding implied assignments, which all have sublevel 3, cause a conflict again in (c). Since assignments of the current decision level that have been inserted before $x_{18}$ have sublevel 2 , the current decision level is divided into two sublevels. This guarantees that the conflict graph of the current sublevel has a unique root; thereby, an ordinary first UIP scheme can be simply applied by considering a sublevel as a decision level.

A conflict clause obtained by using this approach may contain multiple literals that are below the current sublevel, yet those at the same decision level. Literals in that conflict clause with NULL antecedents are necessary to avoid rediscovery of solutions and cannot be removed if they exist; however, it is expected that other literals will be reduced further. This observation leads to another first UIP scheme that is described next.

4.2.3. Decision Level-Based First UIP Scheme*. Here, we present an alternative first UIP scheme that does not require sublevels. Our scheme can be accomplished with small modifications. Traverse a conflict graph from $\kappa$ in the reverse order of implications in a breadth first manner in the same way as an ordinary first UIP scheme, and construct a conflict clause. The main difference is as follows. When the current vertex in the conflict graph is a nondecision assignment with a NULL antecedent, our scheme adds the negation of the literal representation of that vertex to a conflict clause and continues the traversal until a first UIP is found.

Algorithm 4 has the pseudocode for our scheme. The variable, $v$, holds the current vertex in a conflict graph, $G=(V, E)$. The set, $N_{v}$, consists of vertices $u \in V$ such that there is an arc from $u$ to $v$. Vertices selected as the current vertex thus far are stored in $S$. To determine the next vertex, vertices in $N_{v}$ are inserted into $T$ and vertices of the current decision level, $d l$, are stored in $T_{d l}$, by which $T_{d l} \backslash S$ holds candidates for the next vertex to be selected. Note that $T_{d l} \supseteq S$ always holds. Since the traversal is done in the reverse order of implications, $v$ is updated so that it is the one that has most recently been registered among vertices in $T_{d l} \backslash S$. A major characteristic of our

\footnotetext{
${ }^{8}$ As indicated in lines 3-5 of Algorithm 7, the backtracking method we used for this example does not always perform BT. This will be explained later.
} 


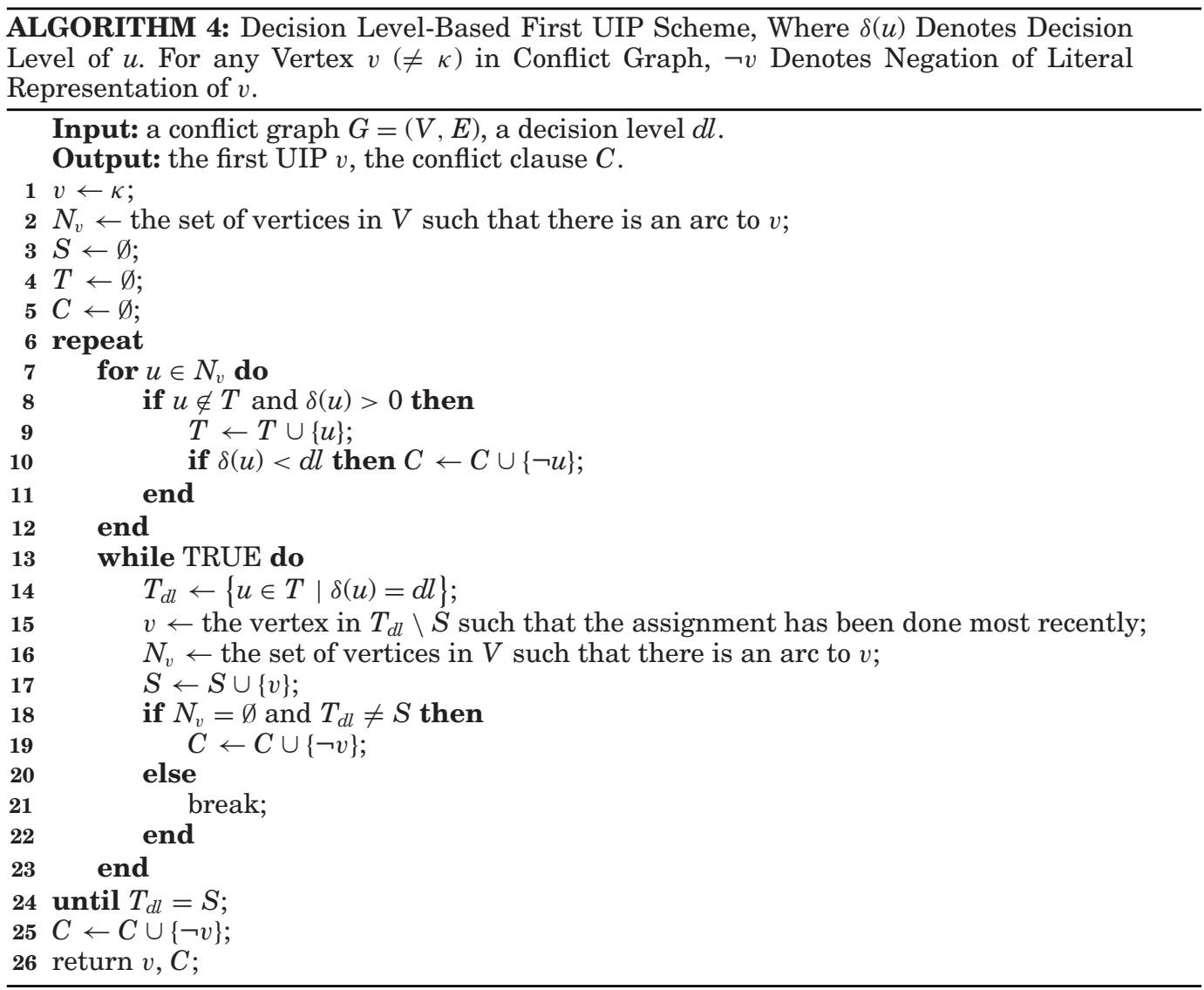

scheme is that $N_{v}$ may be empty. Since $T_{d l}=S$ means that $v$ is a UIP, and $N_{v}=\varnothing$ means that $v$ has no incoming arc, our scheme iterates the update of $v, N_{v}$, and $S$ until $N_{v} \neq \emptyset$ or $T_{d l}=S$. If $T_{d l}=S$ holds for the first time, then $v$ is the first UIP. In this case, the negation of the literal representation of $v$, denoted by $\neg v$, is added to $C$ because $v$ represents the current assignment to the underlying variable of $v$. This is done at line 25 because line 19 is not executed.

Compared to the sublevel-based first UIP scheme, the decision-level-based scheme might be considered to be better. First, it is simpler. Second, a conflict clause contains a unique literal from the current decision level except for those with NULL antecedents. However, it should be noted that conflict clauses obtained by the decision-level-based scheme are not necessarily smaller since the unique implication point is further from $\kappa$ and thus a conflict clause may contain more literals below the current decision level.

Example 4.7. Continuing from Example 4.5, let us consider the case of conflict (c) in Figure 4. Figure 5 illustrates the difference between the two schemes: the sublevelbased scheme finds $x_{6}$ as a first UIP and learns $\neg x_{6} \vee \neg x_{9}$ as a conflict clause, while the decision-level-based scheme finds $\neg x_{20}$ as a first UIP and learns $x_{20} \vee x_{1} \vee \neg x_{18}$ as a conflict clause. Note that the conflict clause in either case does not become a unit clause after backtracking, even though it does not threaten algorithmic correctness because of the flipped decision, $x_{20}$. 


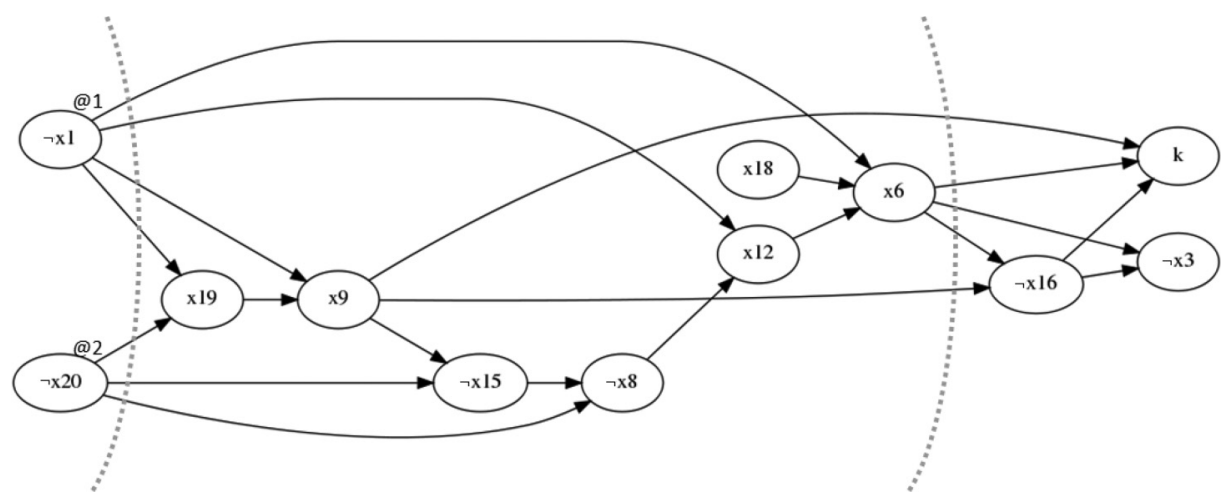

Fig. 5. Implication graph for the case of conflict (c) in Figure 4.

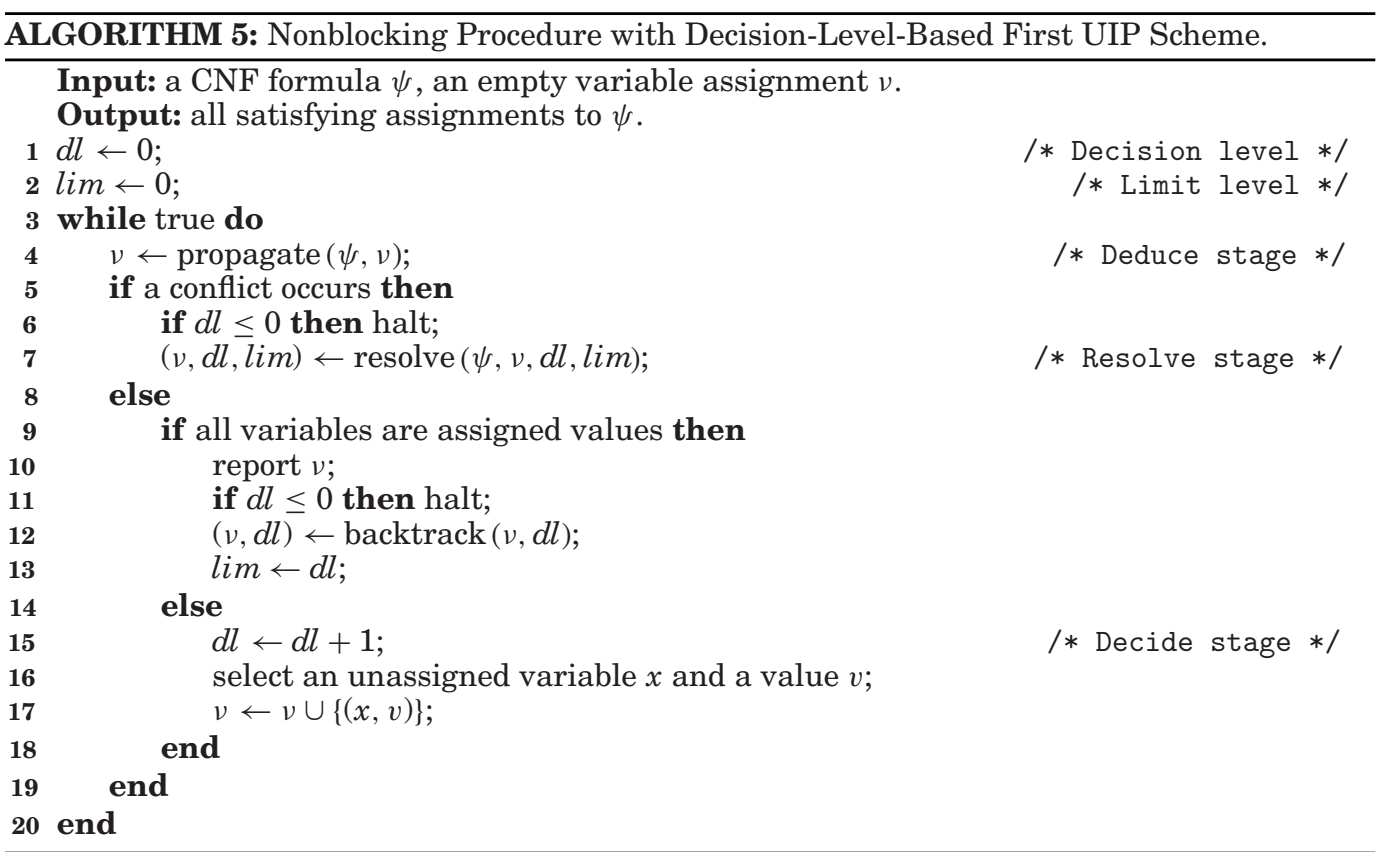

Algorithm 5 is the pseudocode for the nonblocking approach using clause learning with the decision-level-based first UIP scheme. Recall that the function, backtrack, is used to backtrack chronologically, following Algorithm 3. The function, resolve, denotes a generic function for conflict resolution. There are several choices. A simple way of achieving the resolve stage is to undertake clause learning, based on one of the two first UIP schemes and then perform BT. More elaborate methods will be introduced later, with which resolve can also be replaced. Since the variable, lim, is used in one of those methods, it will be introduced when needed.

4.2.4. Conflict-Directed Backjumping. Grumberg et al. augmented a nonblocking approach with conflict resolution by means of a variant of backjumping [Grumberg et al. 2004]. Their backtracking method can be considered as a kind of conflict-directed backjumping ( $C B J$ for short). CBJ has been studied as one of the tree search algorithms 


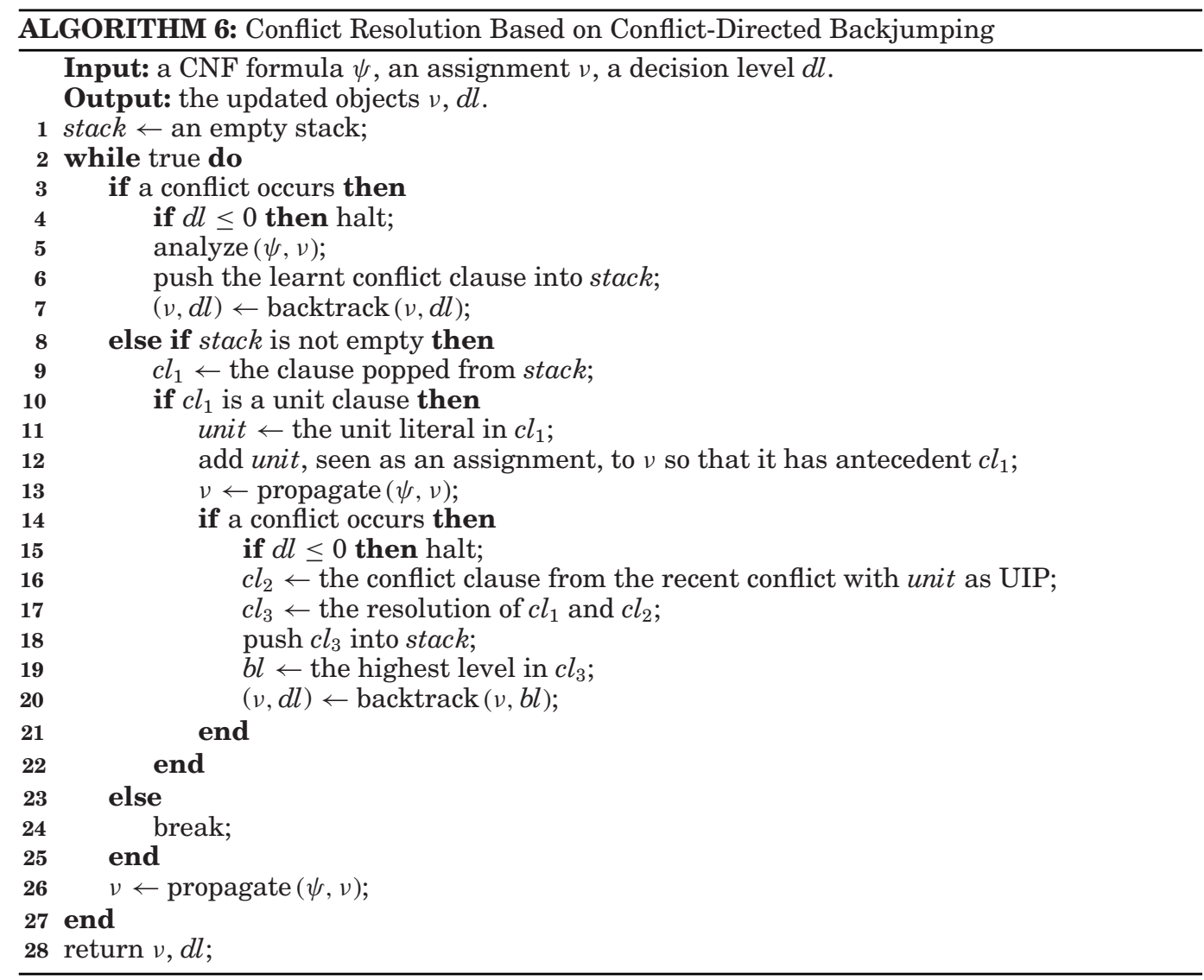

available for constraint satisfaction problems [Prosser 1993; Chen and van Beek 2001; Dechter and Frost 2002].

Algorithm 6 has the pseudocode for CBJ. It consists of chronological backtracking (lines 3-7) and nonchronological backtracking parts (lines 8-22). One of the chronological backtracking parts, the nonchronological backtracking part, and the other part (line 24), where CBJ terminates, is executed, and then unit propagation is performed (line 26) in each iteration of the while loop. Each part is described in the following.

A conflict clause is learnt in the chronological backtracking part by either a sublevelbased or decision-level-based first UIP scheme, which is pushed into the stack, denoted by stack, and then BT is performed. Since BT adds a flipped decision to the current assignment, $v$, other assignments may be implied in the succeeding unit propagation. The chronological backtracking part is iterated (and learnt conflict clauses are piled on stack) until the unit propagation performed at the end of the while loop does not cause a conflict.

The nonchronological backtracking part immediately backjumps to a lower level, if possible, by computing implications from clauses stored in stack. Let $c l_{1}$ be the clause popped from stack. Note that although $c l_{1}$ is a conflict clause learnt previously, it may not be a unit clause since it may be satisfied by flipped decisions or contain several undefined literals. If it is not a unit clause, no implication can be deduced, the succeeding unit propagation does nothing, and the nonchronological backtracking part is selected again (if stack is still not empty). Suppose that $c l_{1}$ is a unit clause. The 


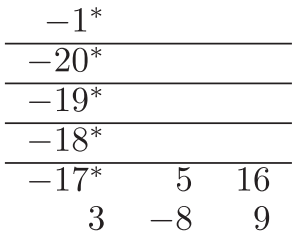

(a)

\begin{tabular}{rrr}
$-1{ }^{*}$ & & \\
\hline$-20^{*}$ & & \\
\hline$-19^{*}$ & & \\
\hline$-18^{*}$ & $17_{*}$ & -11 \\
14 & 6 & -15 \\
-5 & -8 & 4 \\
9 & -16 & -13
\end{tabular}

(b)

\begin{tabular}{rrr}
$-1^{*}$ & & \\
\hline$-20^{*}$ & & \\
\hline$-19^{*}$ & $18_{*}$ & 4 \\
-2 & -5 & 14 \\
-10 & 17 & -15 \\
-13 & &
\end{tabular}

(c)

\begin{tabular}{rrr}
$-1^{*}$ & & \\
\hline$-20^{*}$ & $19 *$ & 10 \\
-5 & -13 & 18 \\
-2 & 14 &
\end{tabular}

(d)

Fig. 6. (a)-(d) Snapshots of nonblocking solver's state in four different cases, indicated in the same way as those in Figure 4. Instance used was uf20-01.cnf, obtained from the Satisfiability Library (SATLIB), and the conflict resolution method used consisted of decision-level-based first UIP scheme and conflict-directed backjumping.

variable, unit, holds the unit literal of $c l_{1}$. This is inserted into the current assignment, $v$, and its implications are propagated at line 13. If a conflict occurs, then a conflict clause, $\mathrm{cl}_{2}$, is computed with a slightly modified version of the sublevel-based first UIP scheme or decision-level-based first UIP scheme such that its UIP is not the first UIP but the unit literal of $c l_{1}$, which can be done simply by continuing search until the target UIP is found. Note that there is a vertex in the conflict graph corresponding to unit because it was inserted in $v$ at line 12 . Since $c l_{2}$ contains the negation of unit, the resolution of $c l_{1}$ and $c l_{2}$ is computed. The resulting clause, $c l_{3}$, is pushed into stack, and nonchronological backtracking is performed at line 20 .

Here, let us remark that the halt at lines 4 and 15 means that the nonblocking procedure is also halted.

Example 4.8. Let us look at Figure 6, which presents snapshots of a nonblocking solver's state, where for the resolve stage, the decision-level-based first UIP scheme and conflict-directed backjumping are used. Since a conflict occurs at the beginning of the while loop in Algorithm 6 in each of the first three cases (a), (b), and (c), the condition at line 3 is satisfied, and a conflict clause is pushed into stack. The function, propagate, is then performed at line 26 . While cases (a) and (b) cause a conflict at this stage, the propagation in case (c) only determines the assignment, $x_{10}$, and it does not cause a conflict. Hence, after case (c), the assignment, v, consists of $\neg x_{1} @ 1, \neg x_{20} @ 2$, $x_{19} @ 2$, and $x_{10} @ 2$, which are determined in this order. The else-if part (lines 8-23) is selected in the next iteration of the while loop because stack holds the three clauses: $\neg x_{5} \vee x_{1} \vee x_{20}, x_{18} \vee x_{20} \vee x_{19} \vee \neg x_{17}$, and $x_{19} \vee x_{20} \vee x_{1} \vee \neg x_{18}$, which are placed from bottom to top in this order. Since the first clause, $c l_{1}=x_{19} \vee x_{20} \vee x_{1} \vee \neg x_{18}$, is already satisfied, $c l_{1}$ is not a unit clause. No propagation takes place because no assignment has been added. The same applies to the next clause, $c l_{1}=x_{18} \vee x_{20} \vee x_{19} \vee \neg x_{17}$. Only the variable, $x_{5}$, is undefined for the last clause, $c l_{1}=\neg x_{5} \vee x_{1} \vee x_{20}$, and hence $c l_{1}$ is a unit clause. The unit literal, $\neg x_{5}$, is inserted into $v$, and unit propagation is then performed. Since the succeeding implied assignments cause a conflict, we compute $c l_{2}$, which is the conflict clause learnt from the conflict analysis with $\neg x_{5}$ as UIP. As a result, we obtain $c l_{2}=x_{5} \vee \neg x_{19} \vee x_{1} \vee x_{20}$. We obtain $c l_{3}=x_{1} \vee \neg x_{19} \vee x_{20}$ by performing the resolution of $c l_{1}$ and $c l_{2}$. Since the highest level in $c l_{3}$ is 2 , we backtrack to level 1 .

4.2.5. Nonchronological Backtracking with Level Limits and lts Combination with $C B J^{\star}$. Here, we present alternative conflict resolution by means of nonchronological backtracking with a backtrack level limit. To the best of our knowledge, this method was first presented by Gebser et al. [2007], although it was within the context of answer set programming. We thus would like to import their idea into our nonblocking procedure. 


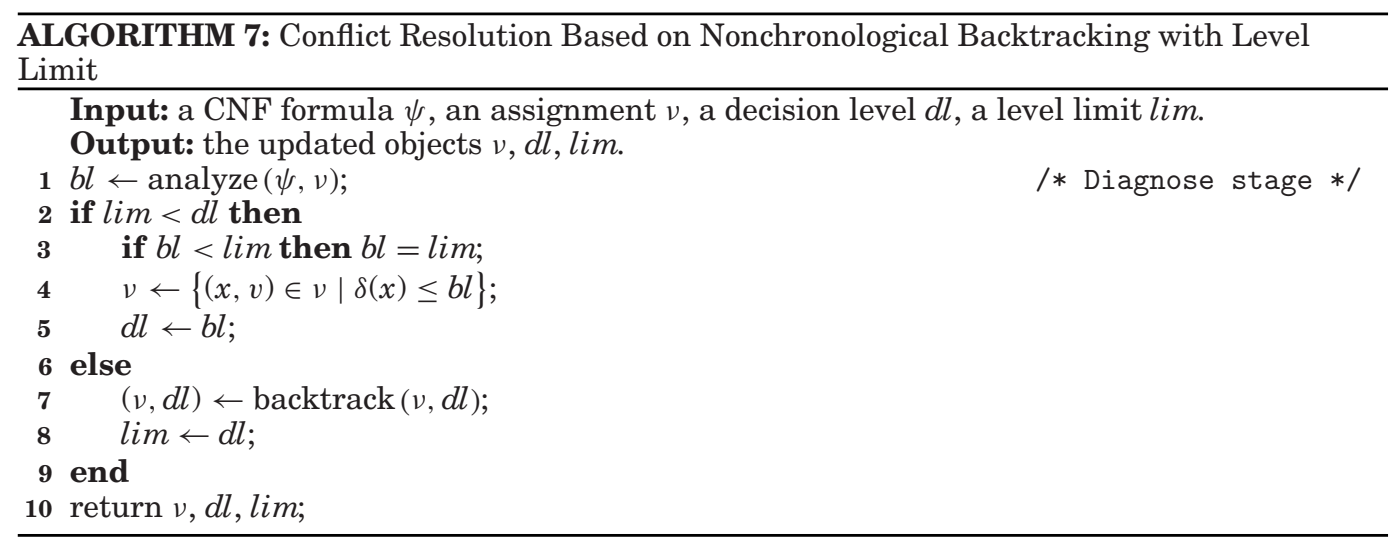

Since the nonblocking procedure does not record blocking clauses, it must not backtrack to an arbitrary level, even though a backtrack level is one that is legitimately derived from a conflict clause. Indeed, nonchronological backtracking without limitations can cancel assignments that have prevented a solver from rediscovering solutions, and those solutions are rediscovered later, causing an infinite loop.

The basic observation here is that nonchronological backtracking can be applied if a backtrack level ensures that solutions are not rediscovered after backtracking. To decide whether the current backtrack level, $b l$, is "safe," we use the variable, lim, that holds the greatest decision level such that there is an enumerated solution with all decision literals up to lim in the current assignment, $v$.

Algorithm 7 provides the pseudocode for our nonchronological backtracking mechanism using a backtrack level limit. We call this approach nonchronological backtracking with a level limit or limited nonchronological backtracking, denoted by BJ, where the underlining means a backtrack level is limited. The variable, lim, is initialized to 0 , and it is updated at line 8 of Algorithm 7 and at line 13 of Algorithm 5, so that it satisfies the property stated previously. That is, since the solution just found in the update part in Algorithm 5 contains all decisions in the current assignment, lim is updated to the decision level after BT is performed. Since lim for the update part in Algorithm 7 is always less than or equal to $d l$, the chronological backtracking part (lines 6-8) is performed if and only if lim $=d l$ holds. Clearly, an enumerated solution that makes lim equal $d l$ must contain the highest decision in the current assignment, $v$. Since BT cancels that decision, lim must be updated to $d l-1$.

Algorithm 7 consists of the nonchronological backtracking part (lines 3-5) and the chronological backtracking part (lines 7-8). Nonchronological backtracking is performed in the nonchronological backtracking part, but the backtrack level, $b l$, is bounded below by lim. If solutions with all decision literals up to lim are exhausted, a solver must backtrack to a lower level than $\lim$ to leave the exhausted search space. If $l i m<d l$ holds, then this means that there may still be solutions to be searched by canceling decision literals of levels above lim. Hence, the nonchronological backtracking part is selected. In the other case, that is, $l i m=d l$, the chronological backtracking part is selected to backtrack to levels lower than lim.

Proposition 4.9. The nonblocking procedure with limited nonchronological backtracking does not find duplicated total satisfying assignments.

Proof. Assume that a solver rediscovers a total satisfying assignment (i.e., solution), $\mu$. Let $t$ be the time when $\mu$ was previously discovered. Let $\lim _{t}$ be $l i m-1$ at time $t$, 
where it is decreased by one because BT is performed just after time $t$. Since time $t$, lim has varied, but $\mathrm{lim}_{t} \leq \mathrm{lim}$ holds. Indeed, if $\mathrm{lim}_{\mathrm{im}}$ reached below $\mathrm{lim}_{t}$ at some time, BT must have been performed at line 7 of Algorithm 7; as stated previously, this means that solutions with all decision literals up to $\mathrm{lim}_{t}$ were all exhausted, and hence a solver would have never searched this exhausted search space afterward due to the presence of flipped decisions. Hence, since time $t$, the $v$ has also varied, but all decision literals up to lim $_{t}$ in $\mu$ have been in $v$. Furthermore, the flipped decision inserted into $v$ by BT after time $t$ has also been in $v$ because it was inserted as a nondecision assignment of level $\lim _{t}$ and it has not been canceled. In particular, the flipped decision must be present in $\mu$. However, this assignment contradicts the highest decision in $\mu$ that was made at time $t$.

We furthermore present a combination of BJ and CBJ. This is obtained by replacing the else part in $\underline{\mathrm{BJ}}$, in which BT is performed, with CBJ: that is,

(1) if lim is less than the current decision level, then perform BJ;

(2) otherwise, perform CBJ.

When CBJ is performed, the variable, lim, is also updated so that after BT is performed (lines 7 and 20 of Algorithm 6), lim is updated to $\mathrm{dl}$ if $\mathrm{lim}$ is larger than the current decision level, $d l$. This approach is denoted by $B J+C B J$.

4.2.6. Implementation. We implemented eight programs based on the nonblocking procedure according to which of the first UIP schemes was selected and which of the conflict resolution methods, that is, BT, BJ, CBJ, or BJ+CBJ, was selected, where BT means BT was performed after clause learning.

\subsection{Formula-BDD Caching Solvers}

4.3.1. Overview. Formula caching refers to a number of techniques to memorize formulas to avoid recomputation of subproblems [Beame et al. 2010]. Examples include a caching technique in probabilistic planning [Majercik and Littman 1998], conflict clauses in SAT [Bayardo and Schrag 1997; Marques-Silva and Sakallah 1999], component caching and other cachings in \#SAT [Bayardo, Jr. and Pehoushek 2000; Bacchus et al. 2003], and blocking clauses in AllSAT [McMillan 2002].

Another type of formula caching in which formulas are associated with concise graph representations of propositional theories such as Free Binary Decision Diagrams (FBDDs), ${ }^{9}$ Ordered Binary Decision Diagrams (OBDDs), and deterministic Decomposable Negation Normal Form $(\mathrm{d}-\mathrm{DNNF})^{10}$ has been studied within the context of knowledge compilation [Huang and Darwiche 2007]. They showed that the trace of an exhaustive DPLL search ${ }^{11}$, which is recorded as a graph, is able to be interpreted as a representation of propositional theory such as FBDD, OBDD, and d-DNNF. Such a representation is able to be efficiently constructed while executing an exhaustive DPLL search. Although exhaustive DPLL search can be used for efficient compilation, compilation in turn can contribute to speeding up exhaustive DPLL search. If a CNF formula is actually compiled into an OBDD, all satisfying assignments can be generated simply

\footnotetext{
${ }^{9} \mathrm{~A}$ free binary decision diagram $(F B D D)$ is a binary decision diagram such that it does not need to be ordered, but no variable appears more than once on any downward path from the root [Knuth 2011, Chapter 7.1.4, p. 261].

${ }^{10} \mathrm{~A}$ negation normal form $(N N F)$ is a propositional formula that is constructed from literals only using conjunction and disjunction, which is represented as a directed acyclic graph such that each internal node is labeled $\wedge$ or $\vee$, and each leaf is labeled a propositional literal or constant. A deterministic decomposable negation normal form ( $d-D N N F)$ is an NNF that satisfies properties called decomposability and determinism [Huang and Darwiche 2007; Darwiche 2001].

${ }^{11}$ DPLL is an algorithmic framework on which modern SAT solvers are based (see Algorithm 1), and an exhaustive DPLL search means an extension of DPLL to finding all solutions.
} 
by traversing all possible paths from the root to the sink node, $T$. Although this seems like taking a long diversion to AllSAT solving, recomputation of many subproblems can be saved due to the caching mechanism. A connection to AllSAT was mentioned in Huang and Darwiche [2007]; however, their primary concern was on the compilation to suitable languages for required queries, and was not restricted to AllSAT. To the best of our knowledge, comparisons have only been conducted between various compilers. An application to AllSAT solving itself was more explicitly mentioned in Toda and Tsuda [2015] and a compilation-based AllSAT solver has been released. However, no comparisons with other AllSAT solvers have yet been conducted and its power remains unknown.

Similar caching techniques appear in other areas such as preimage computation in unbounded model checking [Sheng and Hsiao 2003; Li et al. 2004; Kang and Park 2005], satisfiability [Motter and Markov 2002], discrete optimization [Andersen et al. 2007; Bergman et al. 2016; Hooker 2013], the computation of the Tutte polynomial of a graph with application to network reliability [Sekine et al. 1995; Sekine and Imai 1995; Sekine 1997], generation of combinatorial patterns [Knuth 2011, pp. 249-255, Chapter 7.1.4; Minato 2013], and power distribution network analysis [Inoue et al. 2014, 2015].

This article only deals with a caching method that records pairs of formulas and OBDDs, which we call formula-BDD caching. Formula-BDD caching can be embedded in either blocking or nonblocking procedures. ${ }^{12}$ This is done without almost any loss of optimizations employed in underlying procedures. An exception is where variables must be selected in fixed order at the decide stage. This effect is far from negligible in terms of efficiency, as is well recognized in a single solution to SAT. It has, however, been confirmed in experiments that formula-BDD caching solvers demonstrate a quite good performance on the whole, and provide an efficient method of solution for instances that have a huge number of solutions and cannot possibly be solved by other means.

4.3.2. Caching Mechanism. Here, we provide the basic idea underlying formula-BDD caching by using a simple method of OBDD construction with formula-BDD caching. This method will be elaborated later by implementing it on top of SAT solvers. We assume in OBDD compilation that all variables in a CNF, $\psi$, are ordered in an arbitrary order and assigned indices in increasing order, that is, $x_{i}$ is the $i$-th least variable. We will first introduce terminology.

Definition 4.10. Let $v$ be an assignment such that the $i$-th variable is the least unassigned variable for some $i>1$. A subinstance of a CNF, $\psi$, in $v$ is the CNF formula derived from $\psi$ by applying all assignments up to $x_{i-1}$ to $\psi \cdot{ }^{13}$ The current subinstance refers to the subinstance induced by the current assignment.

Consider the following procedure with a CNF formula, $\psi$, and an empty variable assignment, $v$, as initial arguments.

(1) If a falsified clause exists in the current subinstance, then return $\perp$.

(2) If all variables are assigned values, then return $T$.

\footnotetext{
${ }^{12}$ Only the combination with blocking procedures has been presented in previous work [Huang and Darwiche 2005; Toda and Tsuda 2015].

${ }^{13}$ Since the simple method of OBDD construction we are going to explain does not use unit propagation and all assignments are determined in increasing order of variables, it follows that the subinstance induced by $v$ is the one that can be obtained by applying all assignments defined in $v$ to $\psi$. However, this is no longer the case when OBDD construction is performed on top of SAT solvers since unit propagation may deduce assignments to larger variables than the least unassigned variable. As shown in Proposition 4.17, the basic idea of speeding up OBDD compilation relies on subinstances induced by less variables than the least unassigned variables, not by all assigned variables, and hence we in advance introduce a more general definition here.
} 
(3) $i \leftarrow$ the least index of an unassigned variable.

(4) $f_{0} \leftarrow$ the result obtained by a recursive call with $\psi$ and $v \cup\left\{\left(x_{i}, 0\right)\right\}$.

(5) $f_{1} \leftarrow$ the result obtained by a recursive call with $\psi$ and $v \cup\left\{\left(x_{i}, 1\right)\right\}$.

(6) Return a node with label $i$, the references to LO child $f_{0}$ and to HI child $f_{1}$.

Since different assignments can induce subinstances that are logically equivalent, we exploit dynamic programming to speed up the procedure. To do this efficiently, it is necessary to quickly decide whether the current subinstance is solved. If it is unsolved, we compute an OBDD for all solutions of the current subinstance and memorize it in some data structure such as a hash table, associating it with the current subinstance. Otherwise, the result is obtained as an OBDD from that data structure and recomputation is avoided.

This approach, however, involves an equivalence test of CNF formulas, that is, deciding whether there is a past subinstance such that it is logically equivalent to the current subinstance. Hence, we consider a "weaker" equivalence test. That is, we encode subinstances into formulas so that if the encoded formulas are identical, then these subinstances are logically equivalent. Note that this test is weaker in that logically equivalent subinstances are not required to be encoded into identical formulas. To decide whether the current subinstance is solved, it suffices to search the encoded formula in the set of registered formula-BDD pairs. All requirements for formula-BDD caching to work are simply in the preceding sentence in italics, and any encoding that meets it will do. It should be noted that our test is sound in that acceptance is always a correct decision; however, if we excessively prioritize the efficiency of encoding, logically equivalent subinstances are very likely to result in nonidentical formulas.

Examples of formula-BDD cachings include those of cutsets and separators [Huang and Darwiche 2005], defined next, and a variant of cutsets [Toda and Tsuda 2015].

Definition 4.11. The $i$-th cutset of a CNF formula, $\psi$, is the set of clauses, $C$, in $\psi$ such that $C$ has literals with their underlying variables $x_{j}$ and $x_{k}$ satisfying $j \leq i<k$. The cutwidth of $\psi$ is the maximum size of a cutset of $\psi$.

Definition 4.12. The $i$-th separator of a CNF formula, $\psi$, is the set of variables $x_{j}$ such that some clause in the $i$-th cutset has a literal with its underlying variable $x_{j}$ satisfying $j \leq i$. The pathwidth of $\psi$ is the maximum size of a separator of $\psi$.

Example 4.13. Figure 7 illustrates a CNF formula, where intervals correspond to clauses, and bullets and circles correspond to positive and negative literals. The third cutset consists of $C_{2}$ and $C_{3}$, while the third separator consists of $x_{1}, x_{2}$, and $x_{3}$.

Example 4.14. The diagram on the right in Figure 7 illustrates an OBDD constructed from the CNF $\psi$ on the left, using cutsets as formula-BDD caching. Cutsets are associated with arcs, and satisfied clauses in them are underlined. Look at the two thick paths, which correspond to the following different assignments:

$$
\begin{gathered}
v: x_{1} \mapsto 0, x_{2} \mapsto 0, x_{3} \mapsto 0, \\
\mu: x_{1} \mapsto 1, x_{2} \mapsto 0, x_{3} \mapsto 0 .
\end{gathered}
$$

All variables in $C_{1}$ are assigned values, and $C_{1}$ is satisfied by both $v$ and $\mu$. All variables in $C_{4}$ and $C_{5}$ are unassigned. This means that neither $C_{1}, C_{4}$, or $C_{5}$ affect the equivalence between the two induced subinstances, $\left.\psi\right|_{\nu}$ and $\left.\psi\right|_{\mu}$. In other words, it is sufficient to only consider the other clauses, $C_{2}$ and $C_{3}$, which form the third cutset, to decide whether $\left.\psi\right|_{\nu}$ and $\left.\psi\right|_{\mu}$ are logically equivalent.

Remark 4.15. Since clauses in cutsets and variables in separators are static and do not change during OBDD compilation, we will hereafter assume, without loss of 

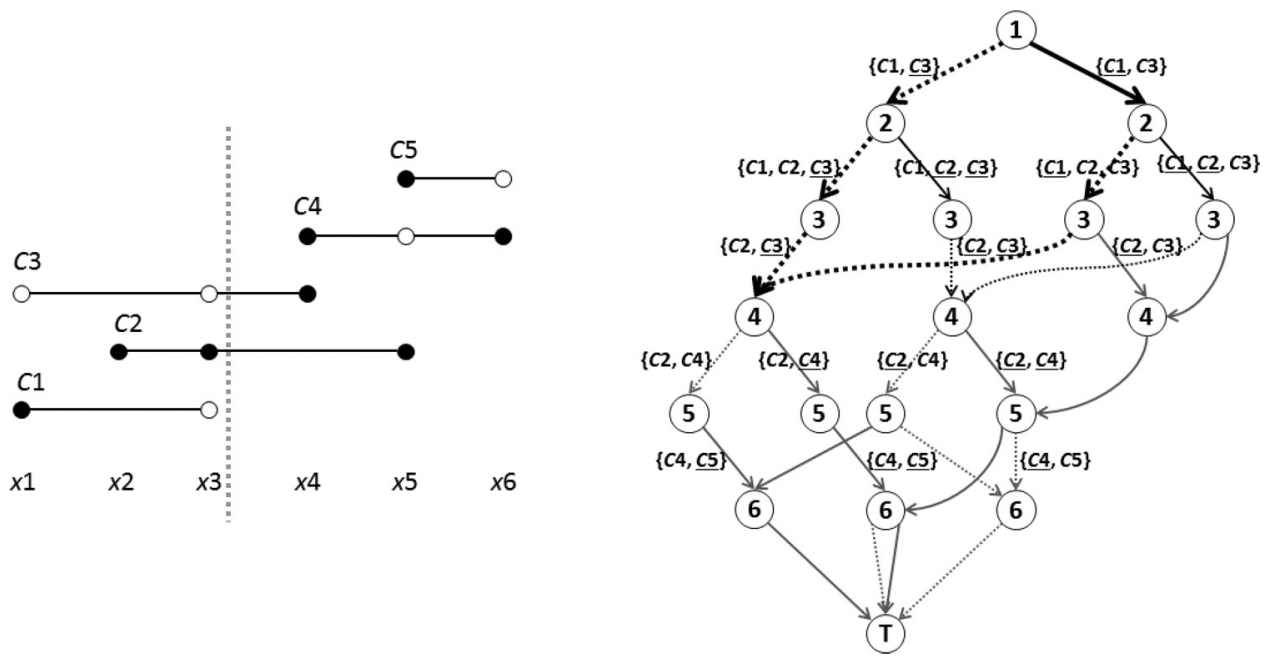

Fig. 7. CNF formula (left) with clauses illustrated by intervals so that bullets are positive literals and circles are negative literals. OBDD for CNF (right), where arcs to sink node $\perp$ are omitted. Cutsets are associated with arcs, where underlined clauses mean they are satisfied. Thick paths are different assignments for CNF. Since they induce logically equivalent CNF, corresponding paths are merged.

generality, that clauses in each cutset and variables in each separator are ordered in fixed order.

We will now introduce auxiliary terminology to clarify the argument.

Definition 4.16. Let $v$ be an assignment such that all of the $i$-th and less variables are assigned values. The state of the $i$-th cutset in $v$ is the bit vector of length equal to the size of the $i$-th cutset such that for each $j$, the $j$-th bit is 1 if the $j$-th clause in the $i$-th cutset is satisfied by $v$, and 0 otherwise. The state of the $i$-th separator in $v$ is the bit vector of length equal to the size of the $i$-th separator such that for each $j$, the $j$-th bit is 1 if the $j$-th variable in the $i$-th separator is assigned value 1 by $v$, and 0 otherwise.

The following proposition states that clauses (and variables) in cutsets (and separators), respectively, meet the requirements of formula-BDD caching. The proof is omitted (see Huang and Darwiche [2005]).

Proposition 4.17. Let $\psi$ be a CNF formula. Let $v$ and $\mu$ be assignments such that the $i$-th variable is the least unassigned variable.

(1) If the state of the $(i-1)$-st cutset in $v$ is identical to the state in $\mu$, then the subinstances of $v$ and $\mu$ are logically equivalent.

(2) If the state of the $(i-1)$-st separator in $v$ is identical to the state in $\mu$, then the subinstances of $v$ and $\mu$ are logically equivalent.

Example 4.18. Continuing from Example 4.14, consider the states of the third cutset, $\left\{C_{2}, C_{3}\right\}$, in $v$ and $\mu$. Since $C_{3}$ is satisfied and $C_{2}$ is not yet satisfied in either assignment, both states are identical. Hence, the paths corresponding to $\nu$ and $\mu$ can be safely merged at a node of label 4 .

An important issue with the formula-BDD caching approach is how to balance quality with efficiency. The quality here refers to how many correct decisions are made. Theoretically, it holds that a correct decision with the separator approach always 


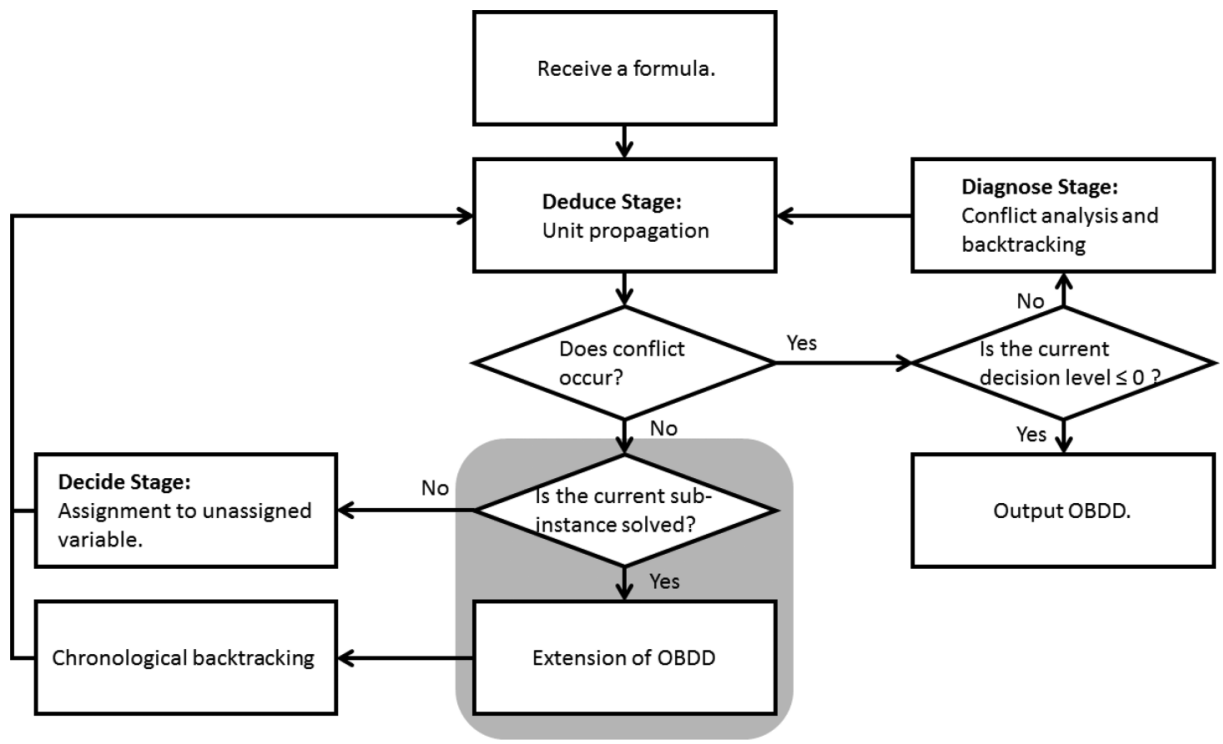

Fig. 8. Flowchart of nonblocking procedure with formula-BDD caching embedded.

implies a correct decision with the cutset approach. Efficiency, on the other hand, refers to how much time is taken to create formulas from subinstances, which substantially amounts to respectively evaluating clauses and variables in cutsets and separators. In terms of efficiency, evaluating clauses in a cutset would require time linear to the total size of clauses due to a lazy evaluation mechanism if it is implemented on top of modern SAT solvers. However, evaluating variables in a separator requires time linear to the number of variables. From the previous argument, we can say that for instances with small cutwidths, the evaluation cost of cutsets is negligible compared to separators and hence cutsets are a better choice, and for instances with many clauses, separators should be used instead.

4.3.3. Embedding Formula-BDD Caching with AlISAT Procedure*. We will now explain how we embed formula-BDD caching with a concrete AllSAT procedure. We will take a nonblocking procedure as an example. To the best of our knowledge, this is the first time that this combination has been presented. The other combination, that is, blocking procedure with formula-BDD caching, is omitted because it was presented [Huang and Darwiche 2005; Toda and Tsuda 2015].

We have thus far assumed that conflict clauses (and blocking clauses in the blocking procedure) are added to a CNF, $\psi$; however, from now on we will assume that they are separately maintained from $\psi$. This leaves $\psi$ unchanged throughout the execution of our AllSAT procedure. Therefore, the cutset and the separator of each level also remain unchanged.

Figure 8 provides a flowchart of nonblocking procedure with formula-BDD caching embedded. The main difference is highlighted in gray. In each iteration of nonblocking procedure, if conflict does not occur, it is decided whether the current subinstance is solved. If the result is true, all solutions that extend the current partial assignment are registered into an OBDD. As shown in Figure 9, this is simply done by adding a path representing the current partial assignment to the root of the subgraph that represents all solutions of the current subinstance, which must exist because the current subinstance was solved in the past and its solutions were registered at that time. In 


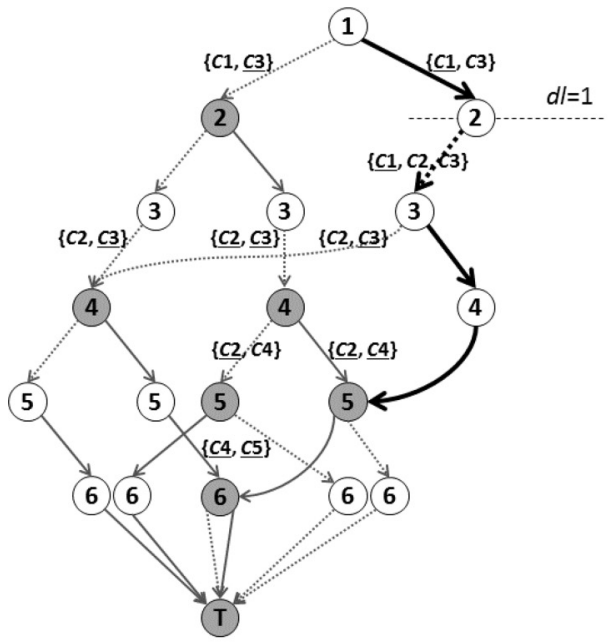

(a)

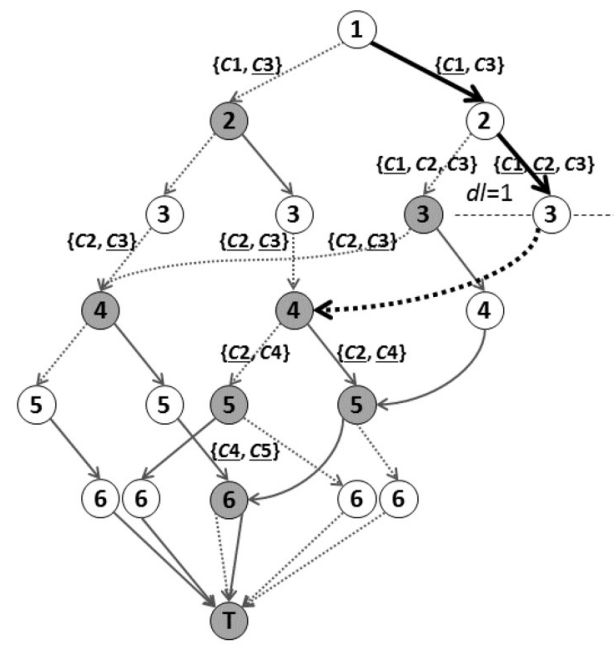

(b)

Fig. 9. Progress in OBDD construction from (a) to (b). Thick arcs in each step represent a path that is about to be added to OBDD under construction, and gray nodes mean that they are recorded and associated with encoded formulas representing their subinstances.

order to efficiently find an OBDD node to which a path is added, we need to maintain pairs of formulas encoded from solved instances and OBDDs representing all solutions of those instances. Such formula-BDD pairs are registered whenever backtracking is performed. This is explained later in detail. An advantage of this approach is that whenever solved subinstances are identified, we can prune search, which has a significant effect on the performance.

Let us look at algorithmic details of how to integrate nonblocking procedure with formula-BDD caching. Algorithm 8 is the pseudocode. The formula-BDD caching mechanism consists of the encode, the extend, and the enroll stages.

At the encode stage, the function, encode, receives a CNF, $\psi$, the current assignment, $v$, and the index, $i-1$, of the one just before the least unassigned variable, $x_{i}$. It computes a formula, $\phi$, for the current subinstance, that is, the subinstance obtained from $\psi$ by applying all assignments up to $x_{i-1}$. We say that $\phi$ is created at $x_{i-1}$ or at level $d l$, where $d l:=\delta\left(x_{i-1}\right)$. We do not present a specific encoding here (see Section 4.3.2 for examples of encoding). If all variables are assigned values, then $i$ is the maximum variable index plus one. In this case, let the function, encode, return 1 , which is the constant formula that evaluates to 1 regardless of assignments. This is why the set, $S$, that holds registered formula-BDD pairs is initialized to $\{(1, \top)\}$. If no variables are assigned values, then $i$ is the least variable index. In this case, let encode return some undefined value, $\phi$, such that no pairs in $S$ have $\phi$ as their first elements.

We search a formula-BDD pair in $S$ at line 15 such that the first element is $\phi$. If it exists, this means that there is a past subinstance such that it is encoded into the same formula, $\phi$, at the same variable, $x_{i-1}$, as the current subinstance. Hence, the current subinstance is already solved, and the resulting OBDD, $g$, is the one associated with the first element, $\phi$. In this case, the function, extend, extends an OBDD, $f$, by adding the path corresponding to the current assignment, $v$, so that the end node of the path is $g$. It returns (the root of) the extended OBDD, $f$, and the array, $\pi$, of OBDD nodes in the path from the root to $g$.

Algorithm 9 has the pseudocode for the function, extend. It traverses the path corresponding to the current assignment, $v$, downward from the root of $f$, add OBDD nodes 


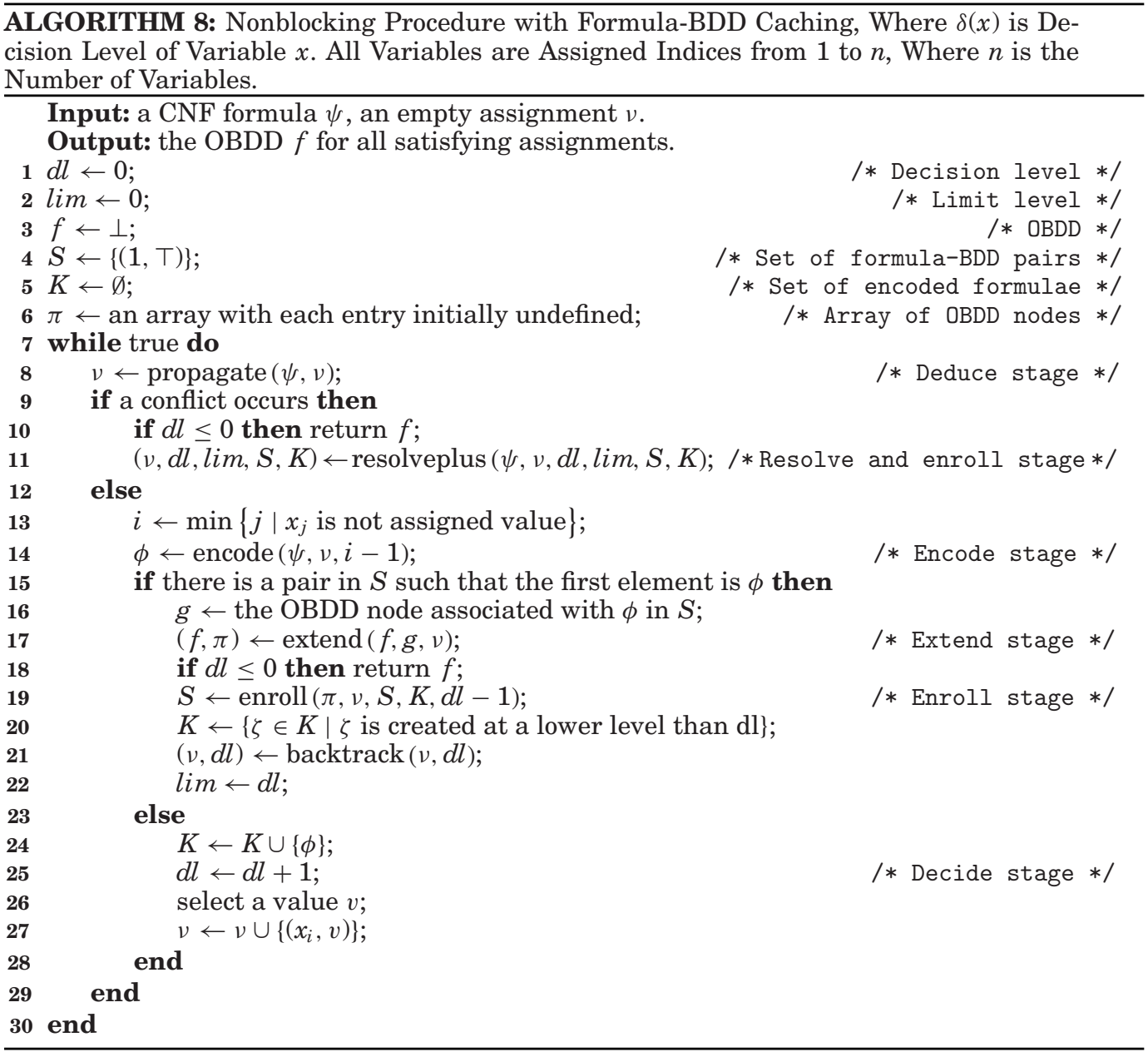

to $f$ if they are not present in $f$, and insert traversed OBDD nodes into the array, $\pi$. The variables, $p$ and $i$, respectively, hold the current OBDD node in the traversal and its variable index. In each iteration of the former while loop (lines 3-11), $p$ is updated according to $v$, and if $p=\perp$ holds, then a loop is terminated. The end condition holds in a finite number of iterations and $v(i)$ is defined until that time because the nonblocking procedure does not rediscover solutions, and hence the whole path corresponding to $v$ is not fully contained in $f$. In each iteration of the latter while loop (lines 15-29), the next node of $p$ is set to the variable, $q$ (lines 16-20), an arc from $p$ to $q$ is connected (lines 21-25), and $p$ is updated to $q$. If the current path is connected to the target node, $g$, then a loop is terminated. An undefined value is then inserted into $\pi$, which is used for the end condition of the while loop in Algorithm 10. Further, $f$ is updated to $\pi$ [1], which is necessary for the case of $f=\perp$.

Example 4.19. Let us look at Figure 9(a). The gray nodes mean that they are recorded in the formula-BDD caching mechanism. Let $f$ be the OBDD under construction outlined in Figure 9(a). Suppose we now have the assignment, $v$, defined as $x_{1} \mapsto 1, x_{2} \mapsto 1$, $x_{3} \mapsto 0$. The third cutset in $v$ consists of $C_{2}$ and $C_{3}$, and both clauses are satisfied in the 


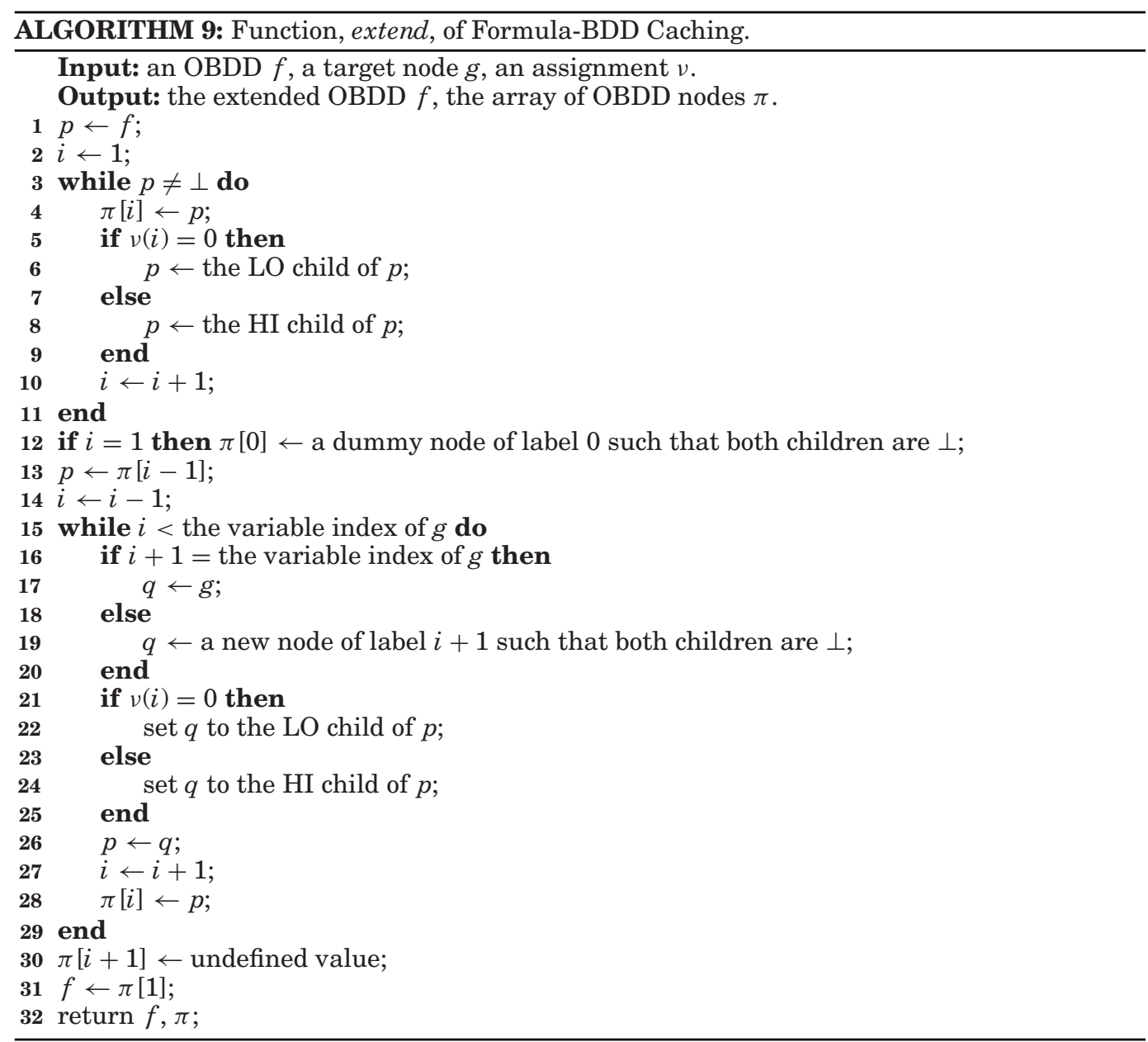

assignments up to $x_{3}$ in $v$. The same state has been registered in the set, $S$, of formulaBDD pairs, as $\left\{C_{2}, C_{3}\right\}$ is associated with an arc from a node of label 3 in Figure 9(a). Hence, the OBDD node, $g$, pointed to by that arc is the target node to which a new path is connected. Let us observe how the OBDD, $f$, is extended. In the former while loop of Algorithm 9, the path, $1 \rightarrow 2$, is traversed. Since the HI child of the end node of that path is not defined, a loop is terminated. In the first iteration of the latter while loop, the end node of that path is set to $p$, and the next node of label 3 is created and set to $q$. An arc from $p$ to $q$ is then connected, and $p$ is updated to $q$. In the next iteration, since the next node is the target node, $g$, an arc from $p$ to $g$ is connected, and a loop is terminated. We finally obtain the extended OBDD outlined in Figure 9(b).

We associate formulas for solved subinstances with the corresponding OBDDs at the enroll stage and insert these formula-BDD pairs into $S$. To do this, an important issue is how to identify when subinstances are solved. We must be careful about this if nonchronological backtracking $(\underline{\mathrm{BJ}})$ is involved because nonchronological backtracking may be performed without exhausting all solutions of subinstances. If formula-BDD pairs for those subinstances are registered in backtracking, then overlooked solutions 


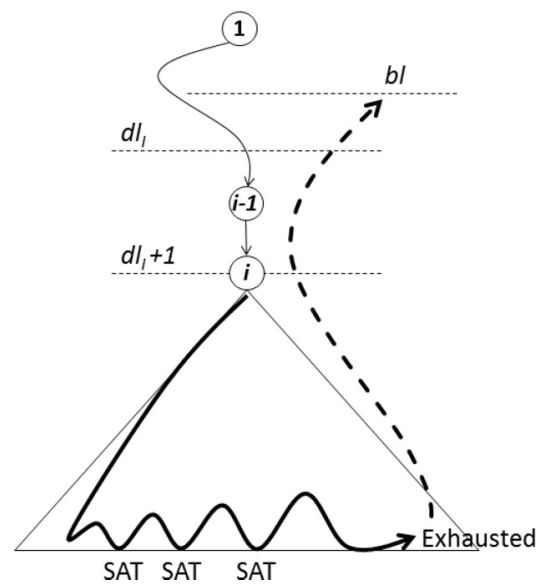

Fig. 10. Scheme for identifying when subinstances are solved. Let $I$ be subinstance induced by assignment such that $i$-th variable is least unassigned variable. $d l_{I}$ denotes decision level of $(i-1)$-st variable, that is, $d l_{I}=\delta\left(x_{i-1}\right)$. Thick curve means exhaustive search for all solutions of $I$, and dashed thick curve means backtracking. If backtrack level $b l$ is less than $d l_{I}$ and at least one solution of $I$ is found, then all solutions of $I$ must be exhausted.

are not searched afterward since formula-BDD pairs prevent a solver from recomputation. This issue is considered in detail in the following (see also Figure 10).

Let $I$ be the subinstance induced by an assignment such that the $i$-th variable is the least unassigned variable. The $d l_{I}$ denotes the decision level of the $(i-1)$-st variable, that is, $d l_{I}=\delta\left(x_{i-1}\right)$. When all solutions of $I$ are found, backtracking to a lower level than $d l_{I}$ is performed to leave the exhausted search space. This is directly triggered by the occurrence of a conflict implying no solution is left or by the discovery of the last solution. Backtracking to a lower level can also be performed without exhausting all solutions; however, this only occurs when no solution of $I$ has yet been found. This is because, otherwise, by definition the variable lim must be larger than $d l_{I},{ }^{14}$ and hence a backtrack level must be at least $d l_{I}$ according to Algorithm 7. Summarizing the preceding, if a backtrack level is less than $d l_{I}$ and at least one solution of I is found, then all solutions of I are exhausted.

Algorithm 10 presents the pseudocode of the function, enroll. The function, enroll, receives an array of OBDD nodes $\pi$, which is the path that has most recently been added; the current assignment, $v$, the set of formula-BDD pairs; $S$, the set of encoded formulas, $K$; and a backtrack level, $b l$.

In each iteration of the while loop, the variable, $p$, holds the current OBDD node in $\pi$ and the variable, $q$, holds the child of $p$ determined by $v$. Note that $q$ may be different from the next entry of $p$ in $\pi$, that is, $\pi[i+1]$, because $v$ is different from the assignment when the most recent path, $\pi$, was added. If $q \neq \pi[i+1]$ holds, then this means that the opposite assignment to $x_{i}$ is implied in $v$ due to backtracking, and hence encoded formulas that correspond to OBDDs in the $(i+1)$-st and larger positions of $\pi$ have been removed from $K$ (line 20 of Algorithm 8). This means that there are no further pairs to be registered.

In the other case, that is, $q=\pi[i+1]$, if $b l<\delta\left(x_{i}\right)$ holds and an encoded formula, $\phi$, was created at $x_{i}$ at some time $t$, then the formula-BDD pair $(\phi, \pi[i+1])$ is registered to $S$ (line 13). These two conditions as well as $q=\pi[i+1]$ guarantee that $x_{i}$ was the one

\footnotetext{
${ }^{14}$ Since unit propagation is performed at the beginning of each repetition, the least unassigned variable $x_{i}$ must be a decision variable, and hence if at least one solution of $I$ is found, then $l i m>d l_{I}$ holds.
} 


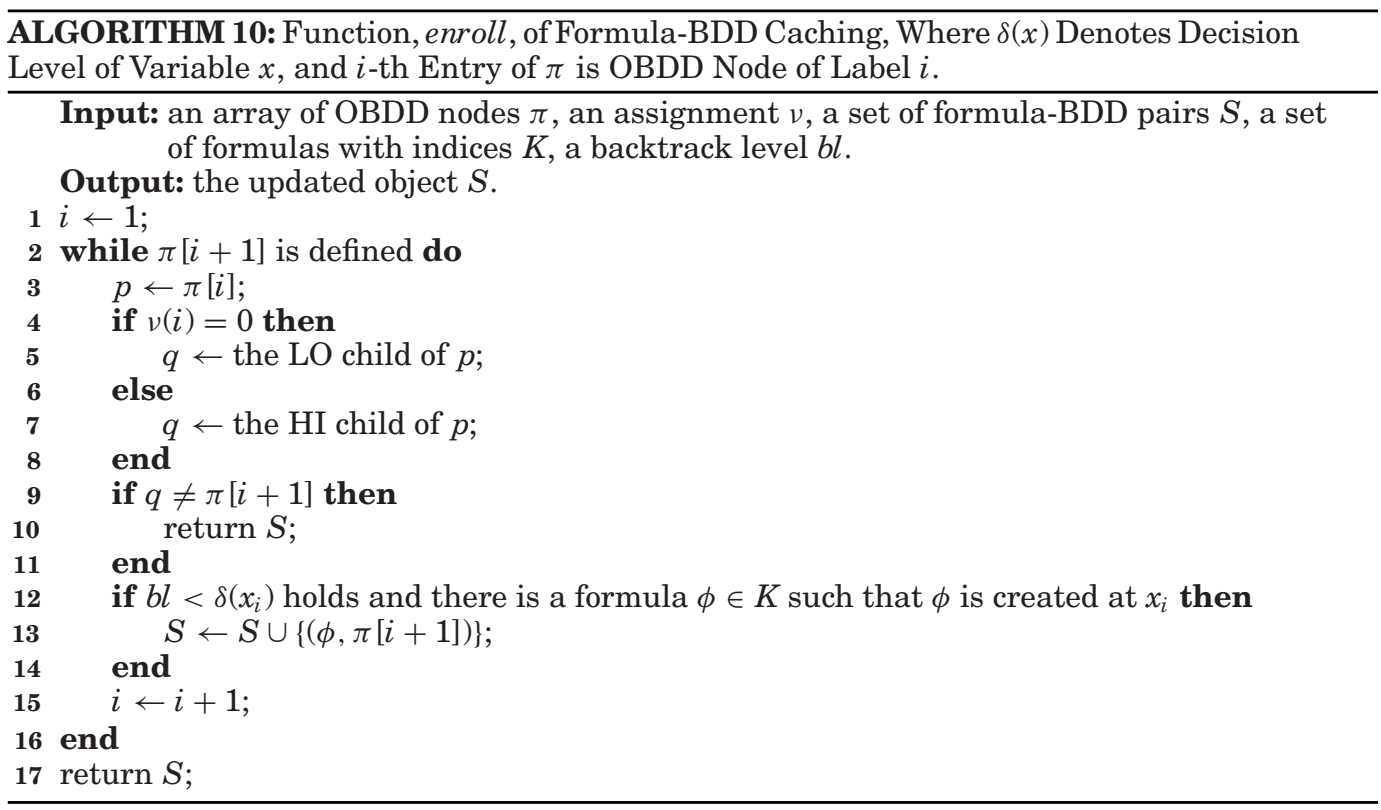

just before the least unassigned variable at time $t$ and that the induced subinstance, $I_{t}$, is now solved. Indeed, let $v_{t}$ be the assignment at time $t$. First, since encoded formulas are only created for the ones just before the least unassigned variables (lines 13 and 14 of Algorithm 8), $x_{i}$ must be such a variable. Second, at least one solution for $I_{t}$ must already be found because $q=\pi[i+1]$ means that a satisfying assignment extending $v_{t}$ is registered in the current OBDD $f$; indeed, since $v\left(x_{j}\right)=v_{t}\left(x_{j}\right)$ holds for all $x_{j} \leq x_{i}$, traverse the path from the root of $f$ according to the assignments in $v_{t}$ up to $x_{i}$; since the current node must be $\pi[i+1]$ due to $q=\pi[i+1]$, further traverse a downward path that leads to $T$. Since we, in addition, have $b l<\delta\left(x_{i}\right)$, the condition for identifying solved subinstances is satisfied (see Figure 10). Clearly, $\pi[i+1]$ holds the OBDD node such that the OBDD rooted by it represents all solutions for $I_{t}$. Therefore, the pair of $\phi$ and $\pi[i+1]$ is inserted into $S$.

Example 4.20. Let us look at Figure 9(a). Let $f$ be the OBDD outlined in Figure 9(a), where suppose that the thick path has not yet been added. Since the subgraph rooted by the LO child of the root of $f$ has been completed, all assignments are canceled and $x_{1}$ is then assigned value 1 because of $B T$. Suppose that $x_{2}$ is assigned value 0 at the decide stage, $x_{3}$ is the least unassigned variable. The function, encode, computes the current cutset state, $\left\{C_{1}, C_{2}, C_{3}\right\}$, which means that $C_{1}$ is satisfied and the other clauses are not yet satisfied by the assignments up to $x_{2}$ in the current assignment. Let $I$ be the current subinstance. Figure 9(a) represents that all solutions of $I$ are exhaustively searched, and the two paths, $1 \rightarrow 2 \rightarrow 3 \rightarrow 4$ and $1 \rightarrow 2 \rightarrow 3 \rightarrow 4 \rightarrow 5$, are added in this order. Before backtracking is performed, the function, enroll, is called with the following arguments: $\pi$ holds the thick path, $v$ represents the corresponding assignment to that path, $S$ consists of the gray nodes associated with cutsets' states, $K$ consists of $\left\{C_{1}, C_{3}\right\}$, $\left\{C_{1}, C_{2}, C_{3}\right\}$, and $\left\{C_{2}, C_{4}\right\}$, and $b l$ holds 0 . Since $\left\{C_{1}, C_{2}, C_{3}\right\}$ was encoded at $x_{2}$ of $\overline{\text { decision }}$ level 1 and the LO child of $\pi$ [2] is equal to $\pi$ [3], the subinstance $I$ turns out to be solved, and the pair of $\left\{C_{1}, C_{2}, C_{3}\right\}$ and $\pi[3]$ is inserted into $S$. As indicated in Figure 9(b), the node stored in $\pi[3]$ is colored in gray. 
A generic function, termed resolveplus, is called at the resolve stage. This function can be replaced with any one of the modified versions of the various conflict resolution methods presented in Section 4.2. The modification is as follows: whenever backtracking is performed in each conflict resolution method, update the set, $S$, of formula-BDD pairs by calling the function, enroll, and then update the set $K$ of encoded formulas in the same way as lines 19 and 20 of Algorithm 8.

4.3.4. Refreshing OBDDs*. A constructed OBDD may be too large to be stored in memory. We present a simple technique to resolve this problem.

Let $n$ be the number of variables. Let $\theta$ be a threshold of OBDD size, where $\theta>n$. Execute the following procedure after an OBDD is extended. If a solution is found and the size of an OBDD is larger than or equal to $\theta-n$, then the current OBDD $f$ is dumped to a file in secondary storage, and all objects $f, S, K$, and $\pi$ of the formula-BDD caching mechanism are refreshed with initial states.

Since our formula-BDD caching mechanism is only used to avoid recomputation of equivalent subproblems, the functionality for refreshing OBDDs never threatens algorithmic correctness, although it may deteriorate performance. Furthermore, the mechanism for avoiding rediscovery of solutions is independent of the formula-BDD caching, and it is actually provided by the exhaustive DPLL search on which formulaBDD caching solvers are based.

We have shown in Proposition 4.9 that the nonblocking solver with limited nonchronological backtracking, BJ, does not rediscover solutions. Since BT and CBJ only perform backtracking to leave exhaustive search space, these types of solvers also do not rediscover solutions. The nonblocking solver with the combination of BJ and CBJ also satisfies this property because each of $B J$ and $C B J$ does. Therefore, if formula-BDD caching solvers are based on either nonblocking solver presented in this article, then they do not rediscover solutions, even though OBDDs are refreshed.

4.3.5. Implementation. We implemented two programs on each nonblocking procedure and two programs on each blocking procedure according to which formula-BDD caching was selected, that is, a cutset or a separator.

\section{EXPERIMENTS}

\subsection{Implementation and Environment}

We implemented all solvers in C on top of MiniSat-C v1.14.1 [Eén and Sörensson 2003], which have been released on our website. ${ }^{15}$ We obtained clasp 3.1.2 from the Potassco project. ${ }^{16}$ Clasp is an actively maintained SAT solver while it is originally developed as an Answer Set Programming (ASP) solver. As a SAT solver, it won first prize at the 2009 and 2011 SAT competitions, and it also provides the CBJ-based AllSAT feature [Gebser et al. 2007, 2009]. As far as we are aware, clasp [Gebser et al. 2007], picosat [Biere 2008], and relsat [Bayardo and Schrag 1997] are the only SAT solvers that support the enumeration of all solutions. Of these, we used clasp for the comparison because it outperformed picosat in a preliminary experiment, and relsat did not support a quiet mode in solution enumeration.

All experiments were performed on a computer with a 2.13-GHz Xeon ${ }^{\circledR} \mathrm{E} 7-2830$ processor and 512GB of RAM, running Red Hat Enterprise Linux 6.3 with gec compiler version 4.4.7. The time limit and memory limit in the execution of each AllSAT solver were set to 600s and 50GB. If either limit was exceeded, the solver was forced to halt.

\footnotetext{
${ }_{15}$ All Solutions SAT Repository by T. Toda, http://www.sd.is.uec.ac.jp/toda/code/allsat.html. It was accessed May 16, 2016. The complete list of used instances in the experiments can be obtained from our website.

${ }^{16}$ Potassco, the Potsdam Answer Set Solving Collection, bundles tools for Answer Set Programming developed at the University of Potsdam, http://potassco.sourceforge.net/. It was accessed Sept. 13, 2015.
} 


\subsection{Solvers}

The types of compared solvers are a blocking solver, a nonblocking solver, a formulaBDD caching solver, and clasp. The first three types have some variations according to which techniques are used (see the end of each subsection in Section 4). Of solvers of the same type, we select a solver with the most solved instances. The three selected solvers, called representative solvers, are as follows.

-Blocking_NoSimple_Cont: a blocking solver with simplification unselected and continuation selected.

-NonBlocking_DLevel_BJ: a nonblocking solver with a decision level first UIP scheme and nonchronological backtracking with a level limit both selected.

-BDD_Cut_NonBlocking_DLevel_BJ: a formula-BDD caching solver with cutset caching selected, which is implemented on top of NonBlocking_DLevel_BJ.

If there are no concerns about confusion, they are abbreviated throughout the section as Blocking, Non-Blocking, and BDD. Notations for the solvers with other configurations are introduced in the same way.

The input and the output for each solver are described in the following. All solvers receive CNF instances in DIMACS CNF format. ${ }^{17}$ Nonblocking solvers and clasp search total satisfying assignments. Since no total assignment is implied by other total assignments, all total satisfying assignments are searched. Hence, their DNFs must mutually coincide. Blocking solvers, on the other hand, search partial satisfying assignments. If simplification of satisfying assignments is disabled, each searched assignment consists of all decision literals, meaning that the assignments to implied variables are uniquely determined. Hence, the searched assignments in this case correspond in a one-to-one way to total assignments, and the number of enumerated assignments must coincide with that of nonblocking solvers and clasp. None of these solvers print out assignments or write them all in a computer file. We simply record the number of assignments to confirm whether solvers find the same number of total assignments and to measure the solvers' performance. For blocking solvers, nonblocking solvers, and clasp, a $\mathrm{CNF}$ instance is solved if searched satisfying assignments represent the same Boolean function as the input CNF.

Formula-BDD caching solvers, on the other hand, construct OBDDs for all total satisfying assignments without explicitly searching those assignments. Constructing OBDDs means implicitly computing all total satisfying assignments because they can be obtained simply by traversing all possible paths from the root to the sink node, $T$. Since listing all assignments from an OBDD is not essential, formula-BDD caching solvers do not output OBDDs or satisfying assignments. We record the number of total satisfying assignments represented by an OBDD for the same reason as stated previously. The computation of counting those assignments can be done in a time linear to the size of an OBDD [Knuth 2011, Algorithm C, Chapter 7.1.4]. The time required for this is not included in the running times of formula-BDD caching solvers. For formulaBDD caching solvers, a CNF instance is solved if a constructed OBDD represents the same Boolean function as the input CNF.

We confirmed that, for each instance, all solvers except for the blocking solvers with simplification enabled computed the same numbers of total satisfying assignments if they were able to solve that instance.

It is known that variable orderings significantly affect the performance of OBDD compilation. Hence, we used the software MINCE version 1.0 [Aloul et al. 2004] to determine static variable order before the execution of formula-BDD caching solvers.

${ }^{17}$ DIMACS Challenge_Satisfiability: Suggested Format, http://www.cs.ubc.ca/ hoos/SATLIB/Benchmarks/ SAT/satformat.ps. It was accessed May 8, 2016. 
Since the execution of MINCE fails for some instances, we used the original order in those cases. The time required for determining variable order was included. Although the time limit is exceeded in preprocessing for some instances, it is negligible for many instances.

\subsection{Problem Instances}

Of the instances available in the repositories listed in the following, we select all instances such that satisfiability is able to be decided in 600 s by either one of the SAT solvers clasp 3.1.2, glucose 4 , minisat 2.2 , or minisat 1.3 , and its result is satisfiable. In the following series of experiments until Section 5.8, we select structured instances. Random instances of SATLIB and SAT Competition 2014 are excluded. The experiments of random instances are described in Section 5.9.

-SATLIB benchmark problems except for random instances (2,736 instances) available in the Satisfiability Library (SATLIB) [Holger and Stützle 2000]. ${ }^{18}$

-SAT Competition 2014 benchmarks, application track (300 instances) and crafted track (300 instances) available in the SAT Competition 2014 Website $^{19}$ (see Belov et al. [2014] for details).

-ISCAS85/89 benchmark circuits in DIMACS CNF format (39 instances), which was converted by the SAT-based test pattern generation system, TG-Pro [Chen and Marques-Silva 2009], from the benchmark circuits provided at 1985 and 1989 International Symposiums on Circuits And Systems (ISCAS). These CNF instances were obtained from the TG-Pro Website. ${ }^{20}$

As a result, we select 2,867 CNF instances. Of these selected instances, satlib denotes those of SATLIB; sc14-app and sc14-crafted denote those of application track and crafted track in SAT Competition 2014; iscas denotes those of ISCAS85/89 benchmark circuits.

Since satlib consists of various instances from different sources, we briefly summarize the description of selected instances for each source (see SATLIB for more details).

- "Flat" Graph Coloring. The instances of this group are encoded from graph coloring problem instances. They are denoted by flat or Flat $x-y$, where $x$ is the number of vertices and $y$ is the number of edges.

- "Morphed" Graph Coloring. The instances of this group also are encoded from graph coloring problem instances. They are denoted by SW100-8- $x$ for $x \in\{0, \ldots, 8, p 0\}$.

-Planning, which was contributed by H. Kautz and B. Selman. The instances of this group are encoded from planning problems in artificial intelligence. The instances for blocks world problems and logistics planning problems are respectively denoted by bw and logistics.

-All-Interval Series, which was contributed by H. Zhang. The instances of this group are encoded from all-interval series problems, which is related to a problem occurring in serial musical composition. They are denoted by ais.

-SAT-encoded Quasigroup (or Latin square) instances, which was contributed by H. Zhang. They are instances encoded from constraints for Quasigroup (or Latin square). They are denoted by $\mathbf{q g}$.

\footnotetext{
${ }^{18}$ SATLIB — The Satisfiability Library by H. Hoos and T. Stützle at Darmstadt University of Technology, http://www.cs.ubc.ca/ hoos/SATLIB/benchm.html. It was accessed May 16, 2014.

${ }^{19}$ SAT Competition 2014, http://www.satcompetition.org/2014/description.shtml. It was accessed Sept. 8, 2015.

${ }^{20}$ TG-Pro-A SAT-based ATPG System by H. Chen and J. Marques-Silva, http://logos.ucd.ie/web/lib/exe/ fetch.php?media=benchmarks:circuit-cnf-tgpro-20110317.tgz. It was accessed May 16, 2014.
} 
-SAT-encoded bounded model checking instances, which was contributed by O. Shtrichman. They are instances encoded by Bounded Model Checker [Biere et al. $1999]^{21}$ from real industrial hardware designs, which was a contribution of the verification technologies department in the IBM Research Laboratory in Haifa (home of RuleBase, the IBM Corporate Model-Checker), and Galileo (a leader in communication hardware design and a heavy user of model checking). They are denoted by bmc.

-DIMACS Benchmark Instances, which were originally provided at the second DIMACS implementation challenge: 1992-1993 [Johnson and Trick 1996]. ${ }^{22}$ These instances comprise the following subgroups.

-Large graph coloring problems, denoted by gcp.

-Instances for learning the parity function, denoted by parity.

-Instances from a problem in inductive inference, denoted by inductive.

-SAT-encoding of Towers of Hanoi, denoted by hanoi.

-Instances from circuit fault analysis, denoted by ssa.

We, in addition, include 63 SAT-encoded instances of frequent itemset mining problems, which are denoted by cp4im, and these instances are available on our website. Here, we explain how we create these CNF instances. We first obtain seven datasets in the data mining domain from the following repository, which are audiology, hepatitis, primary-tumor, zoo-1, heart-cleveland, lymph, and vote. Not all datasets in the repository are used because some datasets are encoded into very large instances.

-CP4IM datasets (16 instances) available in Constraint Programming for Itemset Mining (CP4IM) Website, ${ }^{23}$ which were originally collected from the UCI machine learning repository. ${ }^{24}$

Each line in the datasets is a space-separated list of item identifiers, which is called a transaction, where the last item is removed because it is attribute information. Given a set of transactions, $\mathcal{T}$, and a support, $\theta$, a frequent itemset is a set of items, $S$, such that the number of transactions in $\mathcal{T}$ containing $S$ is larger than or equal to $\theta$, and the frequent itemset mining problem is the problem of generating all frequent itemsets with respect to $\mathcal{T}$ and $\theta$. We first transform the frequent itemset mining problem for each selected dataset, $\mathcal{T}$, and each support, $\theta=\alpha|\mathcal{T}| / 100$ for $\alpha \in\{10,20, \ldots, 90\}$, into propositional Boolean formulas by using the encoding of Guns et al. [2011]. Since these formulas include cardinality constraints, these formulas are then encoded into CNFs with the method of Sinz [2005]. We confirmed that, for each instance, all solvers computed the same numbers of solutions as Linear time Closed itemset Miner (LCM) version $5.3,{ }^{25}$ if they were able to solve that instance, where LCM received not the encoded instances, but the original datasets.

We finally obtain a total of 2,930 CNF instances. Table II indicates the number of selected instances for each group.

\footnotetext{
${ }^{21}$ Bounded Model Checker: BMC by A. Biere, http://www.cs.cmu.edu/ modelcheck/bmc.html. It was accessed May 16, 2016.

${ }^{22}$ The Second DIMACS Implementation Challenge: 1992-1993, Center for Discrete Mathematics \& Theoretical Computer Science Founded as a National Science Foundation Science and Technology Center, http://dimacs.rutgers.edu/Challenges/. It was accessed May 16, 2016.

${ }^{23}$ CP4IM-Constraint Programming for Itemset Mining by T. Guns, https://dtai.cs.kuleuven.be/CP4IM/. It was accessed May 17, 2016.

${ }^{24}$ The UC Irvine Machine Learning Repository at Center for Machine Learning and Intelligent Systems, http://archive.ics.uci.edu/ml/. It was accessed May 17, 2016.

${ }^{25}$ Linear time Closed itemset Miner (LCM) is a well-known itemset miner, and the FIMI'04 "diapers and beer" best implementation award was granted for the LCM implementation version 2 [Uno et al. 2004]. LCM version 5.3 was obtained from http://research.nii.ac.jp/ uno/codes.htm. It was accessed May 31, 2015.
} 


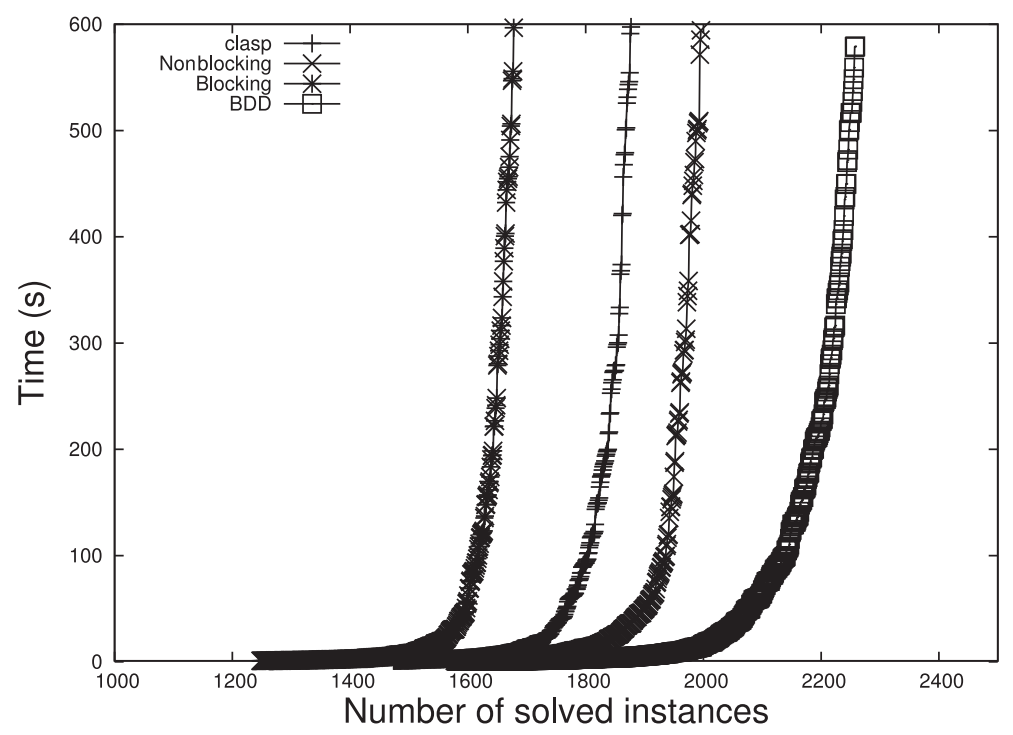

Fig. 11. Cactus plot of representative solvers with respect to running time.

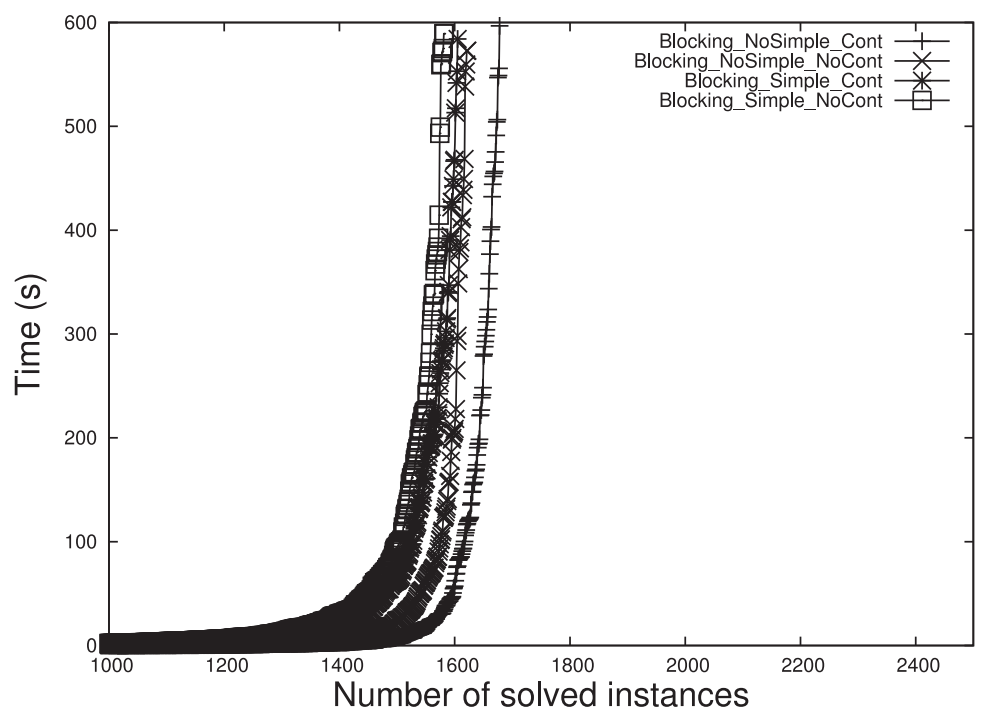

Fig. 12. Cactus plot of blocking solvers.

\subsection{Comparison of Running Times}

Figure 11 is a cactus plot of representative solvers, demonstrating how the number of solved instances increases as the time limit increases. Solved instances are ranked with respect to the times required to solve them. Each point represents a solved instance with its rank (the horizontal coordinate) and the required time (the vertical coordinate). Since one wants to solve as many instances as possible within a time limit, it is advantageous for a solver's curve to increase gently. The formula-BDD caching solver clearly outperforms the other solvers. It is then followed by the nonblocking solver, clasp, and the blocking solver in this order.

Figures 12, 13, and 14 plot differences between solvers of the same types. We can observe from Figure 12 that continuation of search is effective yet simplification degrades 


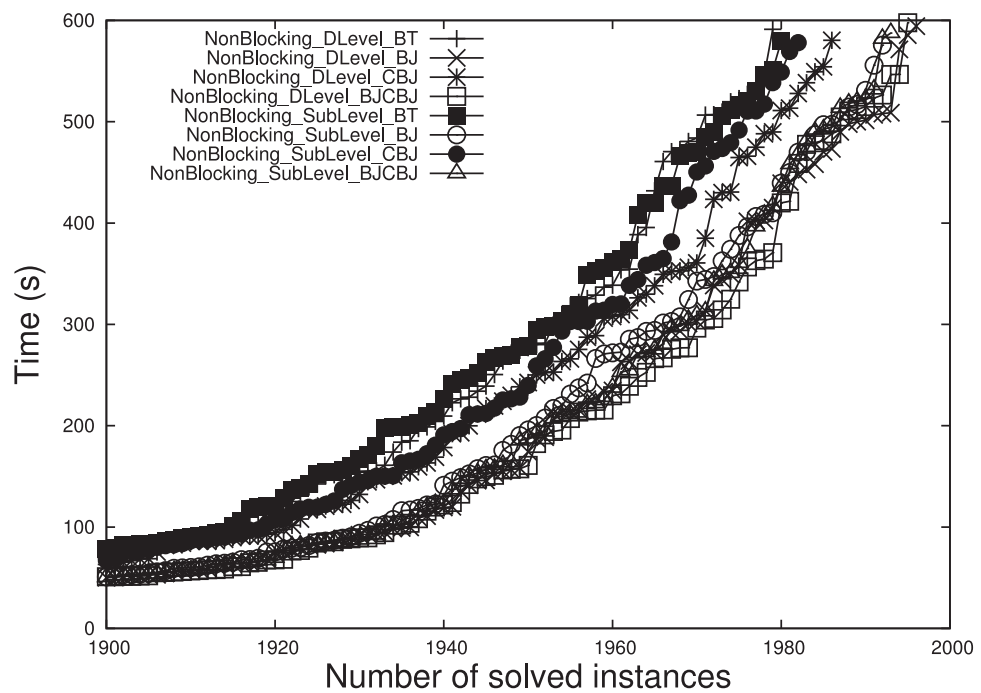

Fig. 13. Cactus plot of nonblocking solvers, where horizontal scale is narrowed to clarify differences.

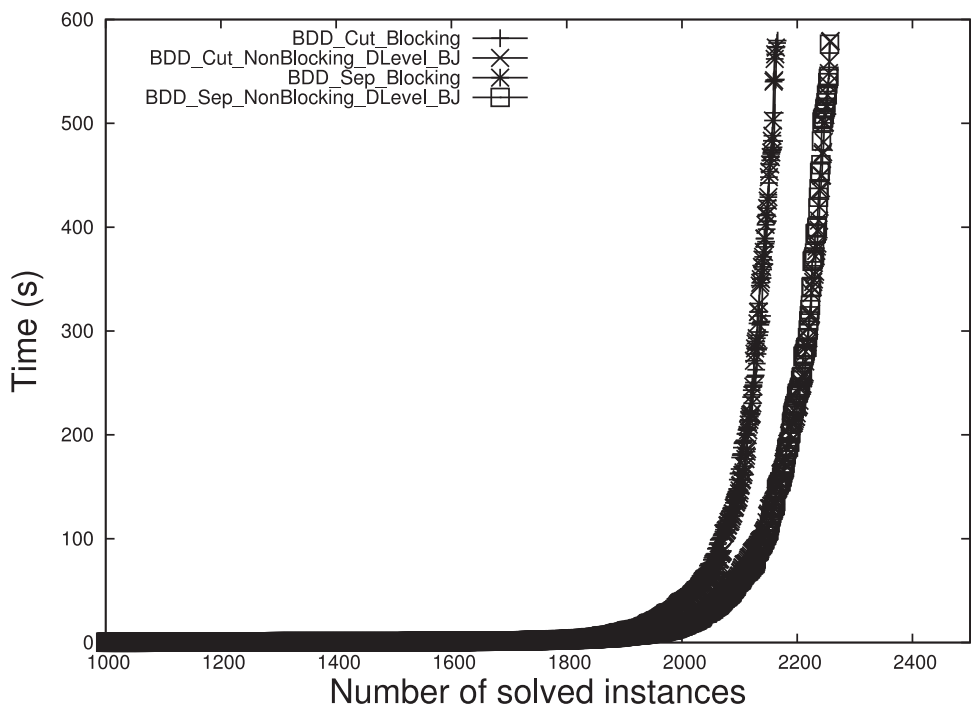

Fig. 14. Cactus plot of formula-BDD caching solvers.

performance. For some instances, simplification enables a solver to find a large number of solutions; however, such instances are limited and the current implementation is not powerful enough to make it possible to solve instances that cannot be handled without simplification. Figure 13 has a narrower horizontal range than the other figures. This is because nonblocking solvers exhibit quite similar performance and they cannot be distinguished otherwise. It is surprising that BT is almost as efficient as the other elaborated backtracking methods. As plotted in Figure 15, clause learning is effective for used instances. We do not present a result over the sublevel-based first UIP scheme because the same result is obtained. This implies that the difference between various backtracking methods themselves is not large. The decision-level-based 


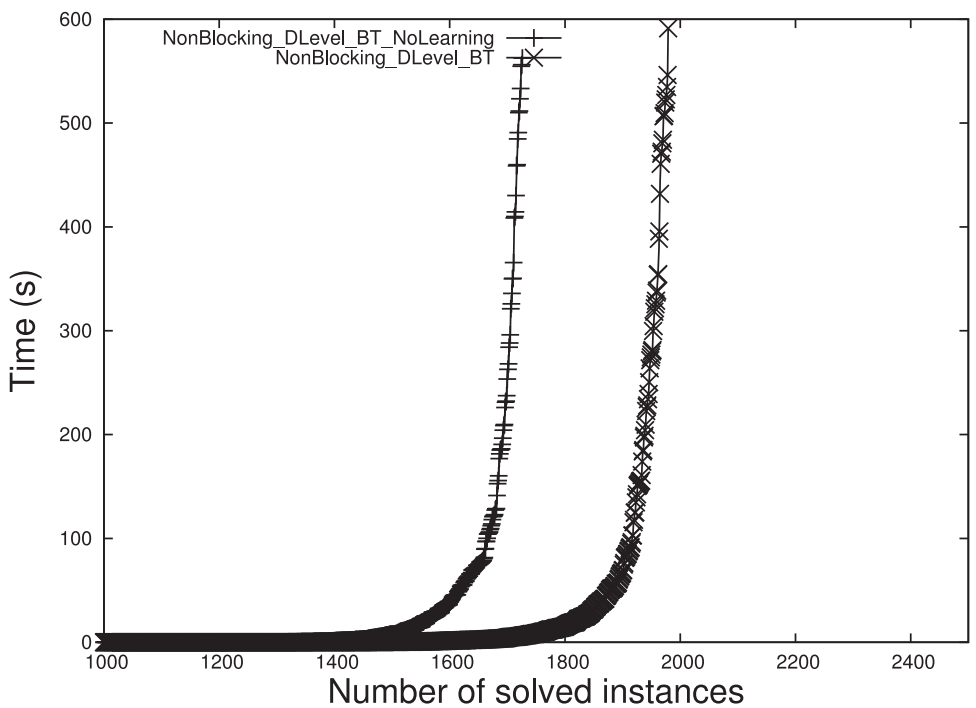

Fig. 15. Cactus plot of nonblocking solvers with clause learning enabled and disabled.

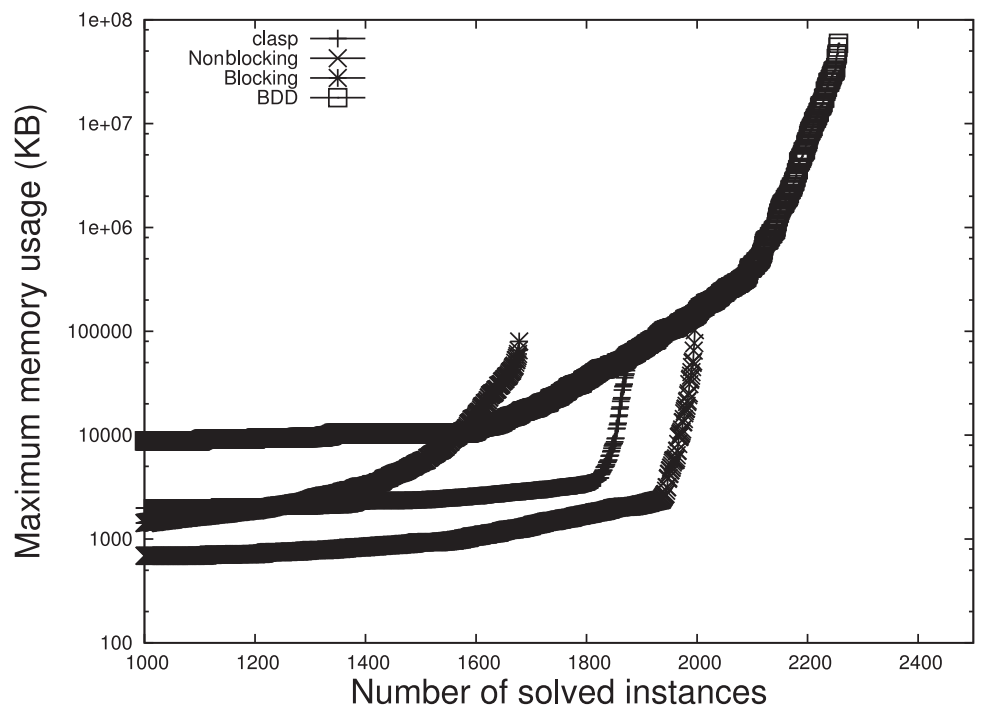

Fig. 16. Cactus plot of representative solvers with respect to maximum memory usage, where vertical axis is on logarithmic scale.

scheme is equal to or more efficient than the sublevel-based scheme. Figure 14 indicates that the nonblocking procedure is clearly better as an underlying solver in which the caching mechanism is embedded, while there is almost no difference between the caching methods.

\subsection{Comparison of Maximum Memory Usage}

Figure 16 is a cactus plot of the maximum memory usage. Solved instances are in turn ranked with respect to the maximum memory usage. Each point then represents a solved instance with its rank (the horizontal coordinate) and the required memory (the 
Table I. Distribution of Solved Instances with Respect to Number of Solutions

\begin{tabular}{l|cccc}
\hline & Blocking & Clasp & Nonblocking & BDD \\
\hline$\left(0,10^{1}\right)$ & 47 & 45 & 48 & 45 \\
{$\left[10^{1}, 10^{2}\right)$} & 20 & 20 & 20 & 18 \\
{$\left[10^{2}, 10^{3}\right)$} & 426 & 426 & 426 & 424 \\
{$\left[10^{3}, 10^{4}\right)$} & 689 & 690 & 690 & 688 \\
{$\left[10^{4}, 10^{5}\right)$} & 413 & 415 & 414 & 412 \\
{$\left[10^{5}, 10^{6}\right)$} & 83 & 138 & 136 & 136 \\
{$\left[10^{6}, 10^{7}\right)$} & 0 & 87 & 83 & 86 \\
{$\left[10^{7}, 10^{8}\right)$} & 0 & 56 & 88 & 91 \\
{$\left[10^{8}, 10^{9}\right)$} & 0 & 0 & 68 & 83 \\
{$\left[10^{9}, 10^{10}\right)$} & 0 & 0 & 23 & 92 \\
{$\left[10^{10}, 10^{11}\right)$} & 0 & 0 & 0 & 83 \\
{$\left[10^{11}, 10^{12}\right)$} & 0 & 0 & 0 & 44 \\
{$\left[10^{12}, 10^{13}\right)$} & 0 & 0 & 0 & 29 \\
{$\left[10^{13}, 10^{14}\right)$} & 0 & 0 & 0 & 19 \\
{$\left[10^{14}, \infty\right)$} & 0 & 0 & 0 & 8 \\
\hline Total & 1,678 & 1,877 & 1,996 & 2,258 \\
\hline
\end{tabular}

vertical coordinate). In terms of memory consumption, the formula-BDD caching solver is the worst, while the nonblocking solver and clasp perform stably. The rapid increase in the curves of the nonblocking solver and clasp is due to the large CNF formulas. Although the formula-BDD caching solver consumes a great deal of memory, it is not unusual for even current laptop computers to have several gigabytes of RAM, and the advantage of the formula-BDD caching solver is not excessively impaired.

\subsection{Comparison of Scalability in Number of Solutions}

As summarized in Table I, each representative solver has the following limit to the number of solutions within the 600 s time limit.

-Blocking: one million solutions.

-Clasp: one hundred million solutions.

-Nonblocking: ten billion solutions.

-BDD: more than one quadrillion solutions.

\subsection{Distribution of Solved Instances over Instance Series}

Table II lists the distribution of solved instances over all instance series. Differences in almost all series in the number of solved instances between solvers can be explained by their scalability.

Exceptions are the sc14-app and sc14-crafted instances. They are clearly harder instances. All solvers are unable to find even one solution in many instances. For sc14-crafted series, solved instances are those with less than 10 solutions with a few exceptions of BDD. Table III summarizes the distribution of all sc14 instances including unsolved instances. Although clasp is able to find relatively many solutions, the other solvers are only able to find less than 10 solutions for a majority of instances. In terms of the ability to find solutions for sc14 instances, the best solver is clearly clasp; the blocking solvers and the nonblocking solvers are a tie, that is, both ranked second. The formula-BDD caching solver is no match for those instances, which is due to fixed variable ordering.

The preferred ranges of instances for representative solvers are outlined in Figure 17 according to the following two factors: hardness of instances and the numbers of solutions instances have. Each solver is placed in such a way that the vertical position 
Table II. Distribution of Solved Instances Over Instance Series, Where Number of Instances in Each Series is Enclosed in Parentheses

\begin{tabular}{|c|c|c|c|c|c|}
\hline & & Blocking & Clasp & Non blocking & BDD \\
\hline ais & (4) & 4 & 4 & 4 & 4 \\
\hline bmc & (13) & 0 & 0 & 0 & 1 \\
\hline bw & (7) & 7 & 7 & 7 & 6 \\
\hline Flat125-301 & (100) & 4 & 51 & 94 & 100 \\
\hline Flat150-360 & (101) & 0 & 12 & 61 & 101 \\
\hline Flat175-417 & (100) & 0 & 2 & 15 & 98 \\
\hline Flat200-479 & (100) & 0 & 0 & 3 & 77 \\
\hline Flat75-180 & (100) & 87 & 100 & 100 & 100 \\
\hline flat & $(1,199)$ & 1,136 & 1,195 & 1,199 & 1,199 \\
\hline gcp & (1) & 0 & 0 & 0 & 0 \\
\hline hanoi & (2) & 2 & 2 & 2 & 2 \\
\hline inductive & (41) & 1 & 5 & 9 & 8 \\
\hline logistics & (4) & 0 & 0 & 0 & 1 \\
\hline parity & (20) & 20 & 20 & 20 & 20 \\
\hline qg & (10) & 10 & 10 & 10 & 10 \\
\hline ssa & (4) & 0 & 0 & 0 & 0 \\
\hline SW100-8-0 & (100) & 0 & 0 & 0 & 0 \\
\hline SW100-8-1 & (100) & 0 & 0 & 0 & 0 \\
\hline SW100-8-2 & (100) & 0 & 0 & 0 & 0 \\
\hline SW100-8-3 & (100) & 0 & 0 & 0 & 0 \\
\hline SW100-8-4 & (100) & 0 & 1 & 5 & 68 \\
\hline SW100-8-5 & (100) & 55 & 100 & 95 & 100 \\
\hline SW100-8-6 & (100) & 100 & 100 & 100 & 100 \\
\hline SW100-8-7 & (100) & 100 & 100 & 100 & 100 \\
\hline SW100-8-8 & (100) & 100 & 100 & 100 & 100 \\
\hline SW100-8-p0 & (1) & 1 & 1 & 1 & 1 \\
\hline sc14-app & (56) & 0 & 0 & 0 & 0 \\
\hline sc14-crafted & (65) & 5 & 3 & 6 & 5 \\
\hline iscas & (39) & 6 & 17 & 19 & 22 \\
\hline cp4im & (63) & 40 & 47 & 46 & 35 \\
\hline Total & $(2,930)$ & 1,678 & 1,877 & 1,996 & 2,258 \\
\hline
\end{tabular}

Table III. Distribution of all sc14 Instances Including Unsolved Instances According to Number of Found Solutions Within Time Limit

\begin{tabular}{l|cccc}
\hline & Blocking & Clasp & Nonblocking & BDD \\
\hline$[0,0]$ & 54 & 22 & 58 & 89 \\
$\left(0,10^{1}\right)$ & 25 & 24 & 22 & 19 \\
{$\left[10^{1}, 10^{2}\right)$} & 8 & 0 & 0 & 0 \\
{$\left[10^{2}, 10^{3}\right)$} & 12 & 1 & 0 & 0 \\
{$\left[10^{3}, 10^{4}\right)$} & 3 & 5 & 1 & 0 \\
{$\left[10^{4}, 10^{5}\right)$} & 4 & 20 & 1 & 0 \\
{$\left[10^{5}, 10^{6}\right)$} & 15 & 27 & 2 & 0 \\
{$\left[10^{6}, 10^{7}\right)$} & 0 & 21 & 0 & 2 \\
{$\left[10^{7}, 10^{8}\right)$} & 0 & 1 & 0 & 0 \\
{$\left[10^{8}, 10^{9}\right)$} & 0 & 0 & 14 & 0 \\
{$\left[10^{9}, 10^{10}\right)$} & 0 & 0 & 23 & 0 \\
{$\left[10^{10}, 10^{11}\right)$} & 0 & 0 & 0 & 0 \\
{$\left[10^{11}, 10^{12}\right)$} & 0 & 0 & 0 & 1 \\
{$\left[10^{12}, 10^{13}\right)$} & 0 & 0 & 0 & 1 \\
{$\left[10^{13}, 10^{14}\right)$} & 0 & 0 & 0 & 0 \\
{$\left[10^{14}, \infty\right)$} & 0 & 0 & 0 & 9 \\
\hline Total & 121 & 121 & 121 & 121 \\
\hline
\end{tabular}




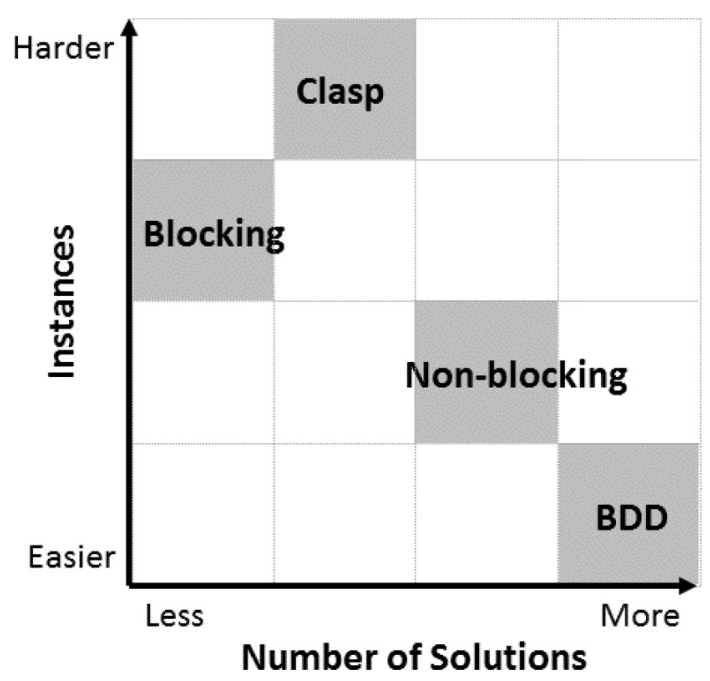

Fig. 17. Preferred ranges of instances for representative solvers.

corresponds to the ability to find solutions for sc14 instances, where the blocking solver is above the nonblocking solver because of greater benefits from SAT solvers, and the horizontal position corresponds to scalability in the number of solutions. Hence, each indicated range refers to instances leftward or downward from it as well as those within it. It should be noted that shrinking the vertical axis would be more suitable for reflecting real performance because the differences between solvers are not very large with respect to the ability of finding solutions for sc14 instances.

\subsection{Comparisons with Publicly Available \#SAT Solvers}

We also conduct a comparison with publicly available \#SAT solvers, that is, sharpSAT version 1.1 [Thurley 2006], c2d version 2.20 [Darwiche 2004], and relsat version 2.20 [Bayardo and Schrag 1997] to evaluate the performance of our developed AllSAT solvers for \#SAT. In the results for the same 2,930 instances, BDD_Cut_NonBlocking_DLevel_BJ solves 2,258, sharpSAT solves 2,227, c2d solves 2,222 , and relsat solves 2,191 . Since relsat supports solution counts, it is used as a solution counter in this comparison. Thus, the formula-BDD caching solver performs better even for \#SAT.

\subsection{Comparisons Over Random Instances}

We also conduct a comparison of running times between representative solvers over random instances. We select all 3,700 uniform random 3 SAT instances, uf (all satisfiable), available in SATLIB. Of all selected instances, Nonblocking solves 3,629, clasp solves 3,585, BDD solves 3,437, and Blocking solves 3,255. As plotted in Figure 18, Nonblocking and clasp outperform BDD. A reason for the degradation of BDD is considered to be a low cache hit rate. Since the previous instances such as Flat and SW have symmetric structures, many equivalent subinstances occur, which favors BDD. However, since random instances do not have such structures, formula-BDD caching has only a limited effect.

\section{CONCLUSION}

We surveyed and discussed major techniques of existing AllSAT solvers. We classified the types of solvers into a blocking solver, a nonblocking solver, and a formula-BDD 


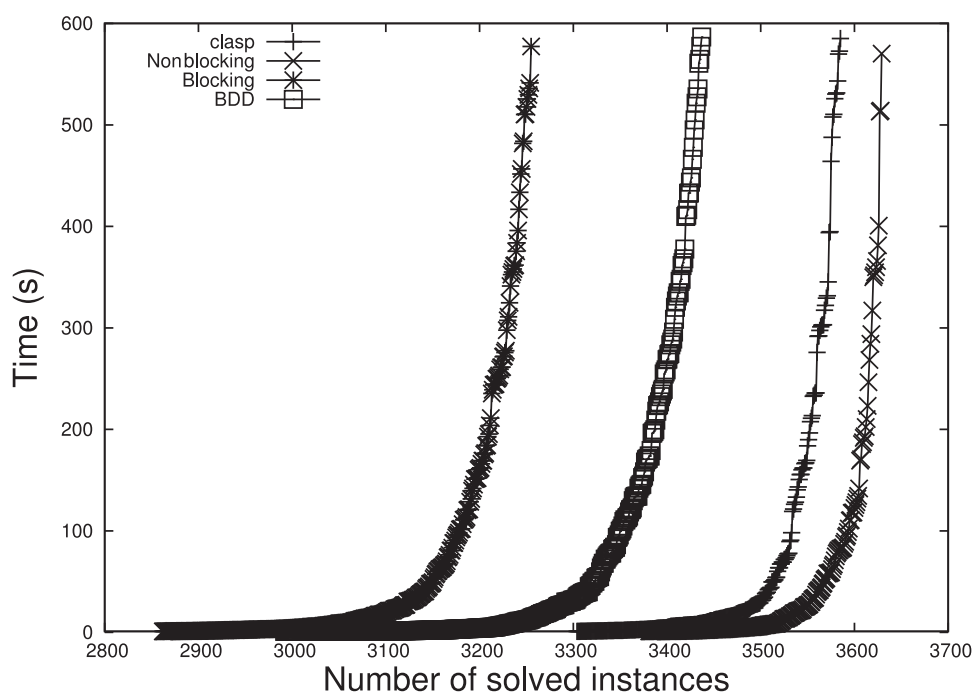

Fig. 18. Cactus plot of representative solvers over random instances.

caching solver. We reliably implemented and released these solvers publicly so that other researchers were able to easily develop their solvers by modifying our code and comparing it with existing methods.

At first, we conducted comprehensive experiments with a total of 2,930 structured instances obtained from the Satisfiability Library (SATLIB), SAT competition 2014, International Symposium on Circuits and Systems (ISCAS), and Constraint Programming for Itemset Mining (CP4IM). Apart from our implemented solvers, we used clasp, which is one of the few off-the-shelf software with solution enumeration support. The experiments revealed three main solver characteristics (600s time limit) (see also Figure 17).

-The formula-BDD caching solver was the most powerful. It provided the most solved instances, including instances with more than one quadrillion solutions. The maximum memory usage amounted to several tens of gigabytes in the worst case, even though it was able to be controlled by refreshing caches at the cost of a low cache hit rate. The formula-BDD caching solver was poor at hard instances due to fixed variable ordering.

-The nonblocking solver was ranked as the next best, followed by clasp. The nonblocking solver and clasp were able to handle instances with ten billion solutions and one hundred million solutions with low maximum memory usage (a few megabytes for the former to several tens of megabytes for the latter), respectively. Although both solvers demonstrated relatively similar performance, the main difference was that for hard instances obtained from the SAT Competition 2014 benchmarks, clasp was good at enumerating solutions on instances which had more than 10 solutions. For those instances, the number of found solutions roughly ranged from $10^{4}$ to $10^{7}$, even though it was not powerful enough to make it possible to solve instances that were not able to be handled by other means.

- The blocking solver was limited to instances with one million solutions as blocking clauses deteriorated the performance of unit propagation. Despite this limitation, the blocking solver still has an advantage that we can use all state-of-the-art (not AllSAT) SAT solvers without any complex modifications, which will fit for finding a small number of solutions of hard instances. 
In addition to structured instances, we also conducted experiments on 3,700 random instances. The formula-BDD caching solver slowed down on these instances because of its low cache hit rate.

We concluded from the preceding that the formula-BDD caching solver was superior in structured instances, while the nonblocking solver and clasp were superior in random instances. However, since not all solutions are necessary in some practical applications and duplicated solutions may be allowed, selecting an appropriate solver is recommended according to the types of instances and applications.

\section{ACKNOWLEDGMENTS}

We would like to thank anonymous referees for carefully reading our manuscript and for giving valuable comments, which resulted in significant improvements. We would also like to thank the reproducibility referee Simon Gog for confirming the reproducibility of our experimental results, which enhanced reliability of our results.

\section{REFERENCES}

Rakesh Agrawal, Tomasz Imieliński, and Arun Swami. 1993. Mining association rules between sets of items in large databases. SIGMOD Rec. 22, 2 (June 1993), 207-216. DOI:http://dx.doi.org/10.1145/ 170036.170072

Rakesh Agrawal and Ramakrishnan Srikant. 1994. Fast algorithms for mining association rules in large databases. In Proceedings of the 20th International Conference on Very Large Data Bases (VLDB'94). Morgan Kaufmann Publishers Inc., San Francisco, CA, 487-499. http://dl.acm.org/citation.cfm?id= 645920.672836

Sheldon B. Akers. 1978. Binary decision diagrams. IEEE Trans. Comput. 27, 6 (June 1978), 509-516. DOI : http://dx.doi.org/10.1109/TC.1978.1675141

Fadi A. Aloul, Igor L. Markov, and Karem A. Sakallah. 2004. MINCE: A static global variable-ordering heuristic for SAT search and BDD manipulation. J. UCS 10, 12 (2004), 1562-1596. DOI : http://dx.doi.org/ 10.3217/jucs-010-12-1562

Henrik Reif Andersen, Tarik Hadzic, John N. Hooker, and Peter Tiedemann. 2007. A constraint store based on multivalued decision diagrams. In Principles and Practice of Constraint Programming-CP 2007. Springer, Berlin, 118-132.

Fahiem Bacchus, Shannon Dalmao, and Toniann Pitassi. 2003. Algorithms and complexity results for \#SAT and Bayesian inference. In Proceedings of the 44th IEEE Annual Symposium on Foundations of Computer Science. IEEE Computer Society, Washington, DC, 340-351. DOI:http://dx.doi.org/ 10.1109/SFCS.2003.1238208

Roberto J. Bayardo, Jr. and Robert C. Schrag. 1997. Using CSP look-back techniques to solve real-world SAT instances. In Proceedings of the 14th National Conference on Artificial Intelligence and 9th Conference on Innovative Applications of Artificial Intelligence (AAAI'97/IAAI'97). AAAI Press, Providence, RI, 203-208. http://dl.acm.org/citation.cfm?id=1867406.1867438

Paul Beame, Russell Impagliazzo, Toniann Pitassi, and Nathan Segerlind. 2010. Formula caching in DPLL. ACM Trans. Comput. Theory 1, 3, Article 9 (March 2010), 33 pages. DOI:http://dx.doi.org/ 10.1145/1714450.1714452

Anton Belov, Daniel Diepold, Marijn J. H. Heule, and Matti Järvisalo (Eds.). 2014. Proceedings of SAT Competition 2014; Solver and Benchmark Descriptions. Department of Computer Science Series of Publications B, Vol. B-2014-2. University of Helsinki, Helsinki. http://hdl.handle.net/10138/135571

David Bergman, Andre A. Cire, Willem-Jan van Hoeve, and John N. Hooker. 2016. Discrete optimization with decision diagrams. INFORMS J. Comput. 28, 1 (2016), 47-66. DOI : http://dx.doi.org/10.1287/ ijoc. 2015.0648

Armin Biere. 2008. PicoSAT essentials. JSAT 4, 2-4 (2008), 75-97. http://jsat.ewi.tudelft.nl/content/ volume4/JSAT4_5_Biere.pdf.

Armin Biere, Alessandro Cimatti, Edmund M. Clarke, and Yunshan Zhu. 1999. Symbolic model checking without BDDs. In Proceedings of the 5th International Conference on Tools and Algorithms for Construction and Analysis of Systems (TACAS'99). Springer-Verlag, London, UK, 193-207. http://dl.acm.org/ citation.cfm?id=646483.691738 
Armin Biere, Marijn Heule, Hans van Maaren, and Toby Walsh. 2009. Handbook of Satisfiability: Volume 185 Frontiers in Artificial Intelligence and Applications. IOS Press, Amsterdam, The Netherlands, The Netherlands.

Jörg Brauer, Andy King, and Jael Kriener. 2011. In Proceedings of the 23rd International Conference on Computer Aided Verification (CAV 2011). Springer, Berlin, 191-207. DOI:http://dx.doi.org/ 10.1007/978-3-642-22110-1_17

Randal E. Bryant. 1986. Graph-based algorithms for boolean function manipulation. IEEE Trans. Comput. C-35, 8 (Aug. 1986), 677-691. DOI : http://dx.doi.org/10.1109/TC.1986.1676819

Huan Chen and Joao Marques-Silva. 2009. TG-PRO: A new model for SAT-based ATPG. In Proceedings of the IEEE International High Level Design Validation and Test Workshop (HLDVT 2009). 76-81. DOI : http://dx.doi.org/10.1109/HLDVT.2009.5340173

Xinguang Chen and Peter van Beek. 2001. Conflict-directed backjumping revisited. J. Artif. Int. Res. 14, 1 (March 2001), 53-81. http://dl.acm.org/citation.cfm?id=1622394.1622397

Edmund Clarke, Daniel Kroening, Natasha Sharygina, and Karen Yorav. 2004. Predicate abstraction of ANSI-C programs using SAT. Formal Meth. Syst. Des. 25, 2-3 (2004), 105-127. DOI:http://dx. doi.org/10.1023/B:FORM.0000040025.89719.f3

Yves Crama and Peter L. Hammer. 2011. Boolean Functions-Theory, Algorithms, and Applications. Encyclopedia of mathematics and its applications, Vol. 142. Cambridge University Press, Cambridge. http://www.cambridge.org/gb/knowledge/isbn/item6222210/?site_locale=en_GB.

Adnan Darwiche. 2001. Decomposable negation normal form. J. ACM 48, 4 (2001), 608-647. DOI : http://dx. doi.org/10.1145/502090.502091

Adnan Darwiche. 2004. New advances in compiling CNF into decomposable negation normal form. In Proceedings of the 16th European Conference on Artificial Intelligence (ECAI'2004), Including Prestigious Applicants of Intelligent Systems (PAIS 2004), Ramon López de Mántaras and Lorenza Saitta (Eds.). IOS Press, Valencia, Spain, 328-332.

Rina Dechter and Daniel Frost. 2002. Backjump-based backtracking for constraint satisfaction problems. Artif. Intell. 136, 2 (2002), 147-188. DOI : http://dx.doi.org/10.1016/S0004-3702(02)00120-0

Niklas Eén and Niklas Sörensson. 2003. An extensible SAT-solver. In Proceedings of the 6th International Conference on Theory and Applications of Satisfiability Testing, (SAT 2003), Selected Revised Papers, Lecture Notes in Computer Science, Vol. 2919, Enrico Giunchiglia and Armando Tacchella (Eds.). Springer, Berlin, 502-518. DOI : http://dx.doi.org/10.1007/978-3-540-24605-3_37

Thomas Eiter, Kazuhisa Makino, and Georg Gottlob. 2008. Computational aspects of monotone dualization: A brief survey. Discrete Appl. Math. 156, 11 (2008), 2035-2049. DOI:http://dx.doi.org/10.1016/ j.dam.2007.04.017. In Memory of Leonid Khachiyan (1952-2005).

Andrew Gainer-Dewar and Paola Vera-Licona. 2016. The minimal hitting set generation problem: Algorithms and computation. CoRR abs/1601.02939 (2016). http://arxiv.org/abs/1601.02939

Malay Kumar Ganai, Aarti Gupta, and Pranav Ashar. 2004. Efficient SAT-based unbounded symbolic model checking using circuit cofactoring. In Proceedings of the IEEE/ACM International Conference on Computer Aided Design (ICCAD'04). IEEE Computer Society, Washington, DC, 510-517. DOI : http://dx.doi.org/10.1109/ICCAD.2004.1382631

Martin Gebser, Benjamin Kaufmann, André Neumann, and Torsten Schaub. 2007. In Proceedings of the 9th International Conference on Logic Programming and Nonmonotonic Reasoning (LPNMR 2007). Springer, Berlin, 260-265. DOI : http://dx.doi.org/10.1007/978-3-540-72200-7_23

Martin Gebser, Benjamin Kaufmann, and Torsten Schaub. 2009. Solution enumeration for projected boolean search problems. In Proceedings of the 6th International Conference on Integration of AI and OR Techniques in Constraint Programming for Combinatorial Optimization Problems (CPAIOR'09). Springer-Verlag, Berlin, 71-86. DOI : http://dx.doi.org/10.1007/978-3-642-01929-6_7

Orna Grumberg, Assaf Schuster, and Avi Yadgar. 2004. In Proceedings of the 5th International Conference on Formal Methods in Computer-Aided Design (FMCAD 2004). Springer, Berlin, $275-289$. DOI : http://dx.doi.org/10.1007/978-3-540-30494-4_20

Tias Guns, Siegfried Nijssen, and Luc De Raedt. 2011. Itemset mining: A constraint programming perspective. Artif. Intell. 175, 1213 (2011), 1951-1983. DOI : http://dx.doi.org/10.1016/j.artint.2011.05.002

Aarti Gupta, Zijiang Yang, Pranav Ashar, and Anubhav Gupta. 2000. Formal Methods in Computer-Aided Design: Third International Conference, FMCAD 2000 Austin, TX, USA, November 1-3, 2000 Proceedings. Springer Berlin Heidelberg, Berlin, Heidelberg, Chapter SAT-Based Image Computation with Application in Reachability Analysis, 391-408. DOI : http://dx.doi.org/10.1007/3-540-40922-X_22

Jiawei Han, Hong Cheng, Dong Xin, and Xifeng Yan. 2007. Frequent pattern mining: Current status and future directions. Data Mining Knowl. Disc. 15, 1 (2007), 55-86. D0I:http://dx.doi.org/ $10.1007 / \mathrm{s} 10618-006-0059-1$ 
Hoos H. Holger and T. Stützle. 2000. SATLIB: An online resource for research on SAT. In Proceedings of the 4th International Conference on Theory and Applications of Satisfiability Testing (SAT 2000), Ian Gent, Hans van Maaren, and Toby Walsh (Eds.), Vol. 63. IOS Press, 283-292.

John N. Hooker. 2013. In Proceedings of the 10th International Conference on Integration of AI and OR Techniques in Constraint Programming for Combinatorial Optimization Problems (CPAIOR 2013). Springer, Berlin, 94-110. DOI : http://dx.doi.org/10.1007/978-3-642-38171-3_7

Jinbo Huang and Adnan Darwiche. 2005. In Proceedings of the 7th International Conference on Theory and Applications of Satisfiability Testing (SAT 2004), Revised Selected Papers. Springer, Berlin, $157-172$. DOI : http://dx.doi.org/10.1007/11527695_13

Jinbo Huang and Adnan Darwiche. 2007. The language of search. J. Artif. Intell. Res. (JAIR) 29 (2007), 191-219. DOI : http://dx.doi.org/10.1613/jair.2097

Takeru Inoue, Keiji Takano, Takayuki Watanabe, Jun Kawahara, Ryo Yoshinaka, Akihiro Kishimoto, Koji Tsuda, Shin-ichi Minato, and Yasuhiro Hayashi. 2014. Distribution loss minimization with guaranteed error bound. IEEE Trans. Smart Grid 5, 1 (Jan. 2014), 102-111. DOI:http://dx.doi.org/ 10.1109/TSG.2013.2288976

Takeru Inoue, Norihito Yasuda, Shunsuke Kawano, Yuji Takenobu, Shin-ichi Minato, and Yasuhiro Hayashi. 2015. Distribution network verification for secure restoration by enumerating all critical failures. IEEE Trans. Smart Grid 6, 2 (March 2015), 843-852. DOI : http://dx.doi.org/10.1109/TSG.2014.2359114

Saïd Jabbour, Jerry Lonlac, Lakhdar Sais, and Yakoub Salhi. 2014a. Extending modern SAT solvers for models enumeration. In Proceedings of the 15th IEEE International Conference on Information Reuse and Integration (IRI 2014). IEEE Computer Society, Washington, DC, 803-810. DOI : http://dx.doi.org/10.1109/IRI.2014.7051971

Saïd Jabbour, Joao Marques-Silva, Lakhdar Sais, and Yakoub Salhi. 2014b. In Proceedings of the 14th European Conference on Logics in Artificial Intelligence (JELIA 2014). Springer International Publishing, Cham, 152-165. DOI : http://dx.doi.org/10.1007/978-3-319-11558-0_11

Saïd Jabbour, Lakhdar Sais, and Yakoub Salhi. 2013. Boolean satisfiability for sequence mining. In Proceedings of the 22nd ACM International Conference on Conference on Information; Knowledge Management (CIKM'13). ACM, New York, NY, 649-658. DOI : http://dx.doi.org/10.1145/2505515.2505577

Karthick Jayaraman, Nikolaj Bjørner, Geoff Outhred, and Charlie Kaufman. 2014. Automated Analysis and Debugging of Network Connectivity Policies. Technical Report MSR-TR-2014-102. Microsoft Research. http://research.microsoft.com/apps/pubs/default.aspx?id=225826.

HoonSang Jin, HyoJung Han, and Fabio Somenzi. 2005. In Proceedings of the 11th International Conference on Tools and Algorithms for the Construction and Analysis of Systems (TACAS 2005), held as part of the Joint European Conferences on Theory and Practice of Software (ETAPS 2005). Springer, Berlin, 287-300. DOI : http://dx.doi.org/10.1007/978-3-540-31980-1_19

HoonSang Jin and Fabio Somenzi. 2005. Prime clauses for fast enumeration of satisfying assignments to boolean circuits. In Proceedings of the 42nd Annual Design Automation Conference (DAC'05). ACM, New York, NY, 750-753. DOI : http://dx.doi.org/10.1145/1065579.1065775

David J. Johnson and Michael A. Trick (Eds.). 1996. Cliques, Coloring, and Satisfiability: Second DIMACS Implementation Challenge, 1993. American Mathematical Society, Boston, MA.

Roberto J. Bayardo, Jr. and Joseph Daniel Pehoushek. 2000. Counting models using connected components. In Proceedings of the 17th National Conference on Artificial Intelligence and 12th Conference on on Innovative Applications of Artificial Intelligence, 2000, Henry A. Kautz and Bruce W. Porter (Eds.). AAAI Press/The MIT Press, Austin, Texas, 157-162. http://www.aaai.org/Library/AAAI/2000/aaai00-024. php.

Hyeong-Ju Kang and In-Cheol Park. 2005. SAT-based unbounded symbolic model checking. IEEE Trans. Comput.-Aided Design Integr. Circ. Syst. 24, 2 (Feb. 2005), 129-140. DOI:http://dx.doi.org/10.1109/ TCAD.2004.841068

Donald E. Knuth. 2011. The Art of Computer Programming: Combinatorial Algorithms, Part 1 (1st ed.). Addison-Wesley Professional, Upper Saddle River, NJ.

Jean-Marie Lagniez and Pierre Marquis. 2016. On preprocessing techniques and their impact on propositional model counting. J. Automat. Reason. (2016), 1-69. DOI:http://dx.doi.org/10.1007/ s10817-016-9370-8

Shuvendu K. Lahiri, Randal E. Bryant, and Byron Cook. 2003. A Symbolic Approach to Predicate Abstraction. Springer, Berlin, 141-153. DOI : http://dx.doi.org/10.1007/978-3-540-45069-6_15

Shuvendu K. Lahiri, Robert Nieuwenhuis, and Albert Oliveras. 2006. SMT Techniques for Fast Predicate Abstraction. Springer, Berlin, 424-437. DOI : http://dx.doi.org/10.1007/11817963_39

C. Y. Lee. 1959. Representation of switching circuits by binary-decision programs. Bell Syst. Tech. J. 38, 4 (1959), 985-999. DOI : http://dx.doi.org/10.1002/j.1538-7305.1959.tb01585.x 
Bin Li, Michael S. Hsiao, and Shuo Sheng. 2004. A novel SAT all-solutions solver for efficient preimage computation. In Proceedings of the 2004 Design, Automation and Test in Europe Conference and Exposition (DATE 2004). IEEE Computer Society, Washington, DC, 272-279. DOI:http://dx.doi.org/ 10.1109/DATE.2004.1268860

Nuno Lopes, Nikolaj Bjørner, Patrice Godefroid, and George Varghese. 2013. Network Verification in the Light of Program Verification. Technical Report. Microsoft Research. http://research.microsoft. com/apps/pubs/default.aspx?id=201589.

Nuno P. Lopes, Nikolaj Bjørner, Patrice Godefroid, Karthick Jayaraman, and George Varghese. 2015. Checking beliefs in dynamic networks. In Proceedings of the 12th USENIX Conference on Networked Systems Design and Implementation (NSDI'15). USENIX Association, Berkeley, CA, 499-512. http://dl.acm.org/citation.cfm?id=2789770.2789805

Stephen M. Majercik and Michael L. Littman. 1998. Using caching to solve larger probabilistic planning problems. In Proceedings of the 15th National Conference on Artificial Intelligence and 10th Innovative Applications of Artificial Intelligence Conference (AAAI'98), Jack Mostow and Chuck Rich (Eds.). AAAI Press/The MIT Press, Madison, Wisconsin, 954-959. http://www.aaai.org/Library/AAAI/ 1998/aaai98-135.php.

Sharad Malik and Lintao Zhang. 2009. Boolean satisfiability from theoretical hardness to practical success. Commun. ACM 52, 8 (Aug. 2009), 76-82. DOI : http://dx.doi.org/10.1145/1536616.1536637

Joao Marques-Silva and Karem A. Sakallah. 1999. GRASP: A search algorithm for propositional satisfiability. IEEE Trans. Comput. 48, 5 (May 1999), 506-521. DOI : http://dx.doi.org/10.1109/12.769433

Ken L. McMillan. 2002. In Proceedings of the 14th International Conference on Computer Aided Verification (CAV 2002). Springer, Berlin, 250-264. DOI : http://dx.doi.org/10.1007/3-540-45657-0_19

Shin-ichi Minato. 2013. Techniques of BDD/ZDD: Brief history and recent activity. IEICE Transactions on Information and Systems 96-D, 7 (2013), 1419-1429. http://search.ieice.org/bin/summary.php?id= e96-d_7_1419.

António Morgado and Joao Marques-Silva. 2005. Good learning and implicit model enumeration. In Proceedings of the 17th IEEE International Conference on Tools with Artificial Intelligence (ICTAI'05). IEEE Computer Society, Washington, DC, 131-136. DOI : http://dx.doi.org/10.1109/ICTAI.2005.69

DoRon B. Motter and Igor L. Markov. 2002. In Proceedings of the 4th International Workshop on Algorithm Engineering and Experiments. Revised Papers. Springer, Berlin, 29-42. DOI:http://dx.doi. org/10.1007/3-540-45643-0_3

Keisuke Murakami and Takeaki Uno. 2014. Efficient algorithms for dualizing large-scale hypergraphs. Discrete Appl. Math. 170 (2014), 83-94. DOI : http://dx.doi.org/10.1016/j.dam.2014.01.012

Knot Pipatsrisawat and Adnan Darwiche. 2007. A lightweight component caching scheme for satisfiability solvers. In Proceedings of the 10th International Conference on Theory and Applications of Satisfiability Testing (SAT 2007), Lecture Notes in Computer Science, Vol. 4501, João Marques-Silva and Karem A. Sakallah (Eds.). Springer, Berlin, 294-299. DOI : http://dx.doi.org/10.1007/978-3-540-72788-0_28

Patrick Prosser. 1993. Hybrid algorithms for the constraint satisfaction problem. Comput. Intell. 9 (1993), 268-299. DOI : http://dx.doi.org/10.1111/j.1467-8640.1993.tb00310.x

Junaid Qadir and Osman Hasan. 2015. Applying formal methods to networking: Theory, techniques, and applications. IEEE Commun. Surv. Tutorials. 17, 1 (First quarter 2015), 256-291. DOI:http:// dx.doi.org/10.1109/COMST.2014.2345792

Kavita Ravi and Fabio Somenzi. 2004. In Proceedings of the 10th International Conference on Tools and Algorithms for the Construction and Analysis of Systems (TACAS 2004), held as part of the Joint European Conferences on Theory and Practice of Software (ETAPS 2004). Springer, Berlin, 31-45. DOI : http://dx.doi.org/10.1007/978-3-540-24730-2_3

Kyoko Sekine. 1997. Algorithm for Computing the Tutte Polynomial and Its Applications. Ph.D. dissertation. Department of Information Science, University of Tokyo.

Kyoko Sekine and Hiroshi Imai. 1995. A Unified Approach via BDD to the Network Reliability and Path Numbers. Technical Report. Department of Information Science, University of Tokyo.

Kyoko Sekine, Hiroshi Imai, and Seiichiro Tani. 1995. In Proceedings of the 6th International Symposium on Algorithms and Computations (ISAAC'95). Springer Berlin, 224-233. DOI:http://dx.doi.org/ 10.1007/BFb0015427

Shuo Sheng and Michael Hsiao. 2003. Efficient preimage computation using a novel success-driven ATPG. In Proceedings of the Conference on Design, Automation and Test in Europe (2003). IEEE Computer Society, Washington, DC, 822-827. DOI : http://dx.doi.org/10.1109/DATE.2003.1253708

Carsten Sinz. 2005. Towards an optimal CNF encoding of boolean cardinality constraints. In Proceedings of the 11th International Conference on Principles and Practice of Constraint Programming (CP 2005), Peter van Beek (Ed.). Springer, Berlin, 827-831. DOI : http://dx.doi.org/10.1007/11564751_73 
Marc Thurley. 2006. In Proceedings of the 9th International Conference on Theory and Applications of Satisfiability Testing (SAT 2006). Springer, Berlin, 424-429. DOI :http://dx.doi.org/10.1007/11814948_38

Takahisa Toda. 2013. In Proceedings of the 12th International Symposium on Experimental Algorithms (SEA 2013). Springer, Berlin, 91-102. DOI : http://dx.doi.org/10.1007/978-3-642-38527-8_10

Takahisa Toda. 2016. Dualization of boolean functions using ternary decision diagrams. Ann. Math. Artif. Intell. (2016), 1-16. DOI : http://dx.doi.org/10.1007/s10472-016-9520-z

Takahisa Toda and Koji Tsuda. 2015. BDD construction for all solutions SAT and efficient caching mechanism. In Proceedings of the 30th Annual ACM Symposium on Applied Computing (SAC'15). ACM, New York, NY, 1880-1886. DOI : http://dx.doi.org/10.1145/2695664.2695941

Takeaki Uno, Masashi Kiyomi, and Hiroki Arimura. 2004. LCM ver. 2: Efficient mining algorithms for frequent/closed/maximal itemsets. In Proceedings of the IEEE ICDM Workshop on Frequent Itemset Mining Implementations (FIMI'04) (CEUR Workshop Proceedings), Roberto J. Bayardo, Jr., Bart Goethals, and Mohammed Javeed Zaki (Eds.), Vol. 126. CEUR-WS.org. http://sunsite.informatik.rwth-aachen.de/ Publications/CEUR-WS/Vol-126/uno.pdf.

Frank van Harmelen, Vladimir Lifschitz, and Bruce Porter. 2007. Handbook of Knowledge Representation. Elsevier Science, San Diego.

Yinlei Yu, Pramod Subramanyan, Nestan Tsiskaridze, and Sharad Malik. 2014. All-SAT using minimal blocking clauses. In Proceedings of the 27th International Conference on VLSI Design and 13th International Conference on Embedded Systems. IEEE Computer Society, Washington, DC, 86-91. DOI : http://dx.doi.org/10.1109/VLSID.2014.22

Shuyuan Zhang, Sharad Malik, and Rick McGeer. 2012. In Proceedings of the 10th International Symposium on Automated Technology for Verification and Analysis. Springer, Berlin, 1-16. DOI:http://dx. doi.org/10.1007/978-3-642-33386-6_1

Received September 2015; revised May 2016; accepted July 2016 